\title{
Who Acquires Information in Dealer Markets?*
}

\author{
Jesper Rüdiger ${ }^{\dagger}$ and Adrien Vigier ${ }^{\ddagger}$
}

\begin{abstract}
We study information acquisition in dealer markets. We first identify a one-sided strategic complementarity in information acquisition: the more informed traders are, the larger market makers' gain from becoming informed. When quotes are observable, this effect in turn induces a strategic complementarity in information acquisition amongst market makers. We then derive the equilibrium pattern of information acquisition and examine the implications of our analysis for market liquidity and price discovery. We show that increasing the cost of information can decrease market liquidity and improve price discovery.
\end{abstract}

JEL classification: D80; D82; G10; G14; G20

Keywords: Dealer Markets; Information Acquisition; Market Liquidity; Price Discovery.

\footnotetext{
${ }^{*}$ We thank Alp Atakan, Tibor Heumann, Stefano Lovo, Larry Samuelson, Peter Norman Sørensen and Xavier Vives for helpful conversations. Any remaining errors are our own.

${ }^{\dagger}$ Department of Business Administration, Universidad Carlos III de Madrid. E-mail: jrudiger@emp.uc3m.es.

${ }^{\ddagger}$ Department of Economics, BI Norwegian Business School. Email: a.h.vigier@gmail.com.
} 


\section{Introduction}

Dealer markets are financial markets in which market makers (MMs) act as intermediaries between sellers and buyers (collectively referred to as traders). The distribution of information among market participants plays a central role in dealer markets, for instance regarding market liquidity. The classic model of dealer markets (Glosten and Milgrom, 1985) assumes that traders have superior information, yet empirical evidence shows that MMs can be better informed than traders. ${ }^{1}$ What does the structure of dealer markets imply about when we should expect to see one situation or the other?

In a dealer market, MMs supply liquidity by quoting bid and ask prices. Traders then submit buy and sell (market) orders. ${ }^{2}$ The literature often distinguishes between two kinds of traders, speculators and liquidity traders: the former trade for profits, and the latter due to liquidity shocks. A MM buys low and sells high, adjusting his bid-ask spread (henceforth spread) to (a) the adverse selection faced and (b) the competition to offer the best quotes. Suppose now that the cost of acquiring information about an asset's value is the same for all market participants, that is, for traders and MMs: who then becomes informed? To address this question, the present paper analyses a simple two-stage game: information acquisition takes place in the first period, and trade in the second.

We first identify a one-sided strategic complementarity in information acquisition. $M M s^{\prime}$ gain from becoming informed is increasing in the probability that traders are informed. The logic is simple. The more informed traders are, the worse the adverse selection facing uninformed MMs, who respond by increasing their spreads. This, in turn, softens price competition for informed MMs, who can now increase their own spreads. So MMs' incremental trading profit from being informed increases with the probability that traders are informed. By contrast, as traders make less profits from trading when MMs are informed than when they are uninformed, traders' gain from becoming informed is always decreasing in the probability that MMs are informed. The result of these observations is a one-sided strategic complementarity in information acquisition.

We then address the question of who acquires information, and show that the microstructure of dealer markets pins down the pattern of information acquisition, as illustrated in Figure 1. If the cost of information and the share of speculators comprising the market are small then

\footnotetext{
${ }^{1}$ Glosten and Milgrom (1985) were primarily interested in understanding the workings of dealer markets in the presence of insider trading. The empirical literature is discussed in Section 7.

${ }^{2}$ The bid price (respectively ask price) is the price at which MMs buy (resp. sell) and traders sell (resp. buy) the asset. The bid-ask spread refers to the difference between the two.
} 


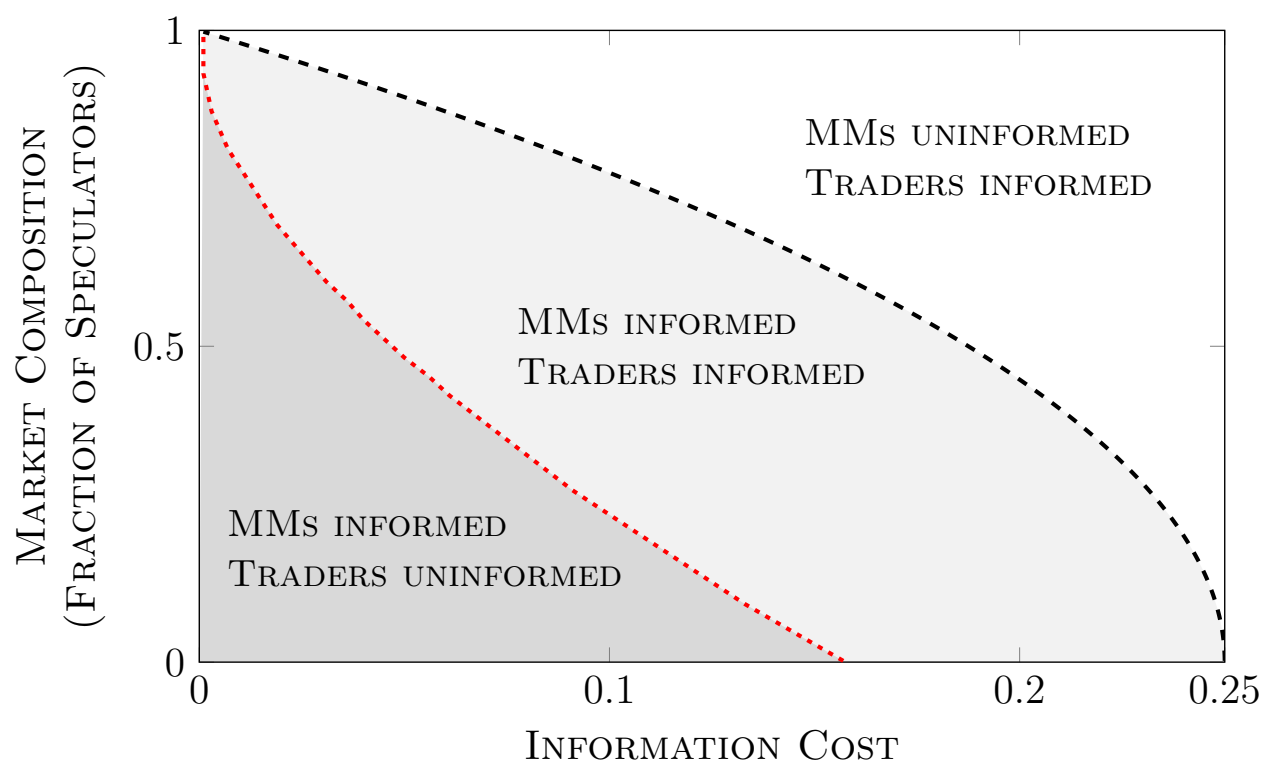

Figure 1: Who Acquires Information

MMs acquire information whereas speculators choose to remain uninformed; if they are large the situation is reversed: speculators acquire information but MMs remain uninformed. ${ }^{3}$ In between, MMs and speculators all become informed with positive probability.

The logic behind who acquires information is as follows. Consider first the effect of information cost. Due to the positive fraction of liquidity traders, a MM's gain from becoming informed remains bounded away from zero as long as not all his competitors acquire information with probability one. As a result, MMs' information acquisition probability is pushed towards one as the information cost tends to zero. An uninformed MM then expects profitable market orders to be picked off by his competitors with probability close to 1 . He therefore faces severe adverse selection, that leads him to set large spreads. This, in turn, implies that the probability that an informed trader finds a profitable trade and the profit that can be made on this trade both go to zero as the cost tends to zero. Traders' gain from acquiring information is therefore second order in the cost of information. In consequence, at small information costs, traders best respond by remaining uninformed.

Consider next larger information costs; to illustrate the main mechanism in this case, suppose that none of the market participants acquire information. As MMs then face no adverse selection, the spreads equal zero. The price at which MMs buy the asset thus equals the price at which traders buy the asset. Similarly, the price at which MMs sell the asset equals

\footnotetext{
${ }^{3}$ The latter case therefore microfounds Glosten-Milgrom types of markets.
} 
the price at which traders sell the asset. So conditional on order execution, an informed trader makes the same profit as an informed MM. However, MMs face execution risk, which traders do not. ${ }^{4}$ So traders' expected profit when informed is larger than MMs'. We show this way that a cost range exists in which MMs are uninformed and speculators acquire information.

The effect of market composition on information acquisition is explained as follows. A speculator abstains from trading when she is uninformed, so MMs only recoup the cost of information from executing the orders of liquidity traders. MMs therefore stop acquiring information when the fraction of speculators in the market becomes sufficiently large. The information acquired by MMs in turn determines speculators' incentives to become informed: speculators remain uninformed when the fraction of liquidity traders is large (in which case most MMs are informed), whereas speculators acquire information when liquidity traders are rare (in which case most MMs are uninformed).

Our analysis shows that, in sharp contrast to models in which MMs are uninformed, increasing the cost of information can decrease market liquidity and improve price discovery. ${ }^{5}$ We furthermore show that an increase in the fraction of speculators can increase market liquidity and reduce price discovery.

We divide for expository purposes the analysis in two parts. We first study a baseline model in which traders submit market orders without having observed MMs' quotes. ${ }^{6}$ This assumption enables us to illustrate the underlying economic principles at work in the simplest possible setting. We then let the traders observe MMs' quotes before placing their market orders. All main results continue to hold, but making the quotes observable yields additional insights. In particular, the mechanism at play in the one-sided strategic complementarity in information acquisition previously highlighted induces in this case a strategic complementarity in information acquisition amongst $M M$ s. Information acquired by one MM now leaks through to the traders via the quotes of that MM, so the more information acquired by any MM the more informed traders are. The rest of the mechanism is as described earlier: more informed trading worsens adverse selection for uninformed MMs which, in turn, softens price competition for informed MMs.

\footnotetext{
${ }^{4} \mathrm{~A}$ market order is executed with probability 1 at the quoted price. By contrast, a quote may not be executed. A quote in our setting corresponds to a limit order, and execution risk plays a key role in limit order markets, where it is a key determinant of a trader's choice between market and limit orders (see, e.g. Kaniel and Liu (2006)).

${ }^{5}$ We use the expected spread to measure (inverse) market liquidity, and the expected squared price error to measure (inverse) price discovery.

${ }^{6}$ One interpretation is that quotes are hidden limit orders, as in e.g. Boulatov and George (2013).
} 
The related literature is discussed in the next paragraphs. Section 2 presents the baseline model, which we analyze in Sections 3 to 5. Section 3 takes the probabilities with which different market participants acquire information as given, and examines the resulting trading game. Section 4 endogenizes information acquisition. Section 5 investigates market liquidity and price discovery. Section 6 extends the baseline model by allowing traders to observe MMs' quotes before market orders are submitted. Section 7 discusses the model and results, and relates our findings to the empirical literature, as well as to recent developments in financial markets.

Related literature. The literature on information acquisition in financial markets stretches back at least to Grossman and Stiglitz (1980) and Verrecchia (1982). Glosten and Milgrom (1985) introduced the model of dealer markets on which we build. Endogenizing traders' information acquisition is relatively straightforward in this model, if one maintains the assumption that MMs are uninformed (see, e.g., Foucault, Pagano and Röell (2013)). By contrast, the problem of information acquisition by MMs is non-trivial. If one fixes traders' information, the problem is formally equivalent to the information acquisition problem in a standard (firstprice sealed-bid common-value) auction setting, analyzed in e.g. Milgrom (1981), Lee (1984), Persico (2000), or Atakan and Ekmekci (2019). The present paper is the first to analyze information acquisition simultaneously occurring on both sides of a dealer market, and to investigate how information acquired by one side of the market affects incentives to acquire information on the other side.

Within the literature on dealer markets, Chamley (2007) allows traders to acquire costly information; Leach and Madhavan (1993), Bloomfield and O'Hara (2000), and de Frutos and Manzano (2005) take on the other hand traders' information as given, and explore MMs' incentives to manipulate prices in order to learn from the order flow. Our paper is also connected to a broader literature on two-sided information acquisition. Dang (2008) analyzes a bargaining game in which the buyer can acquire information before offering a price; the seller observes the offer and can acquire information before deciding whether or not to sell. Unlike our setting, the price-setter is monopolistic and information may be acquired after the price is observed. Tirole $(2009,2015)$ and Bolton and Faure-Grimaud (2010) examine contracting environments in which both parties can acquire information. However, the setting they explore is quite different from ours and offers the players a great deal of commitment power, which is typically lacking in asset markets. We discuss in Section 7 how our paper relates to the literature on limit-order markets, in which market participants choose between 


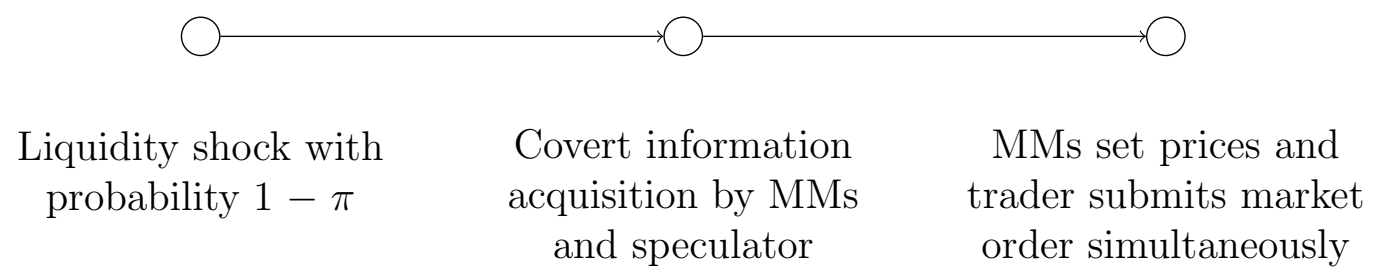

Figure 2: Timing

demanding and supplying liquidity.

\section{Baseline Model}

We consider the market for a risky asset with random value $V$ where $\mathbb{P}(V=1)=\mathbb{P}(V=0)=$ $\frac{1}{2}$. The realization of $V$ is denoted $v$. There are two market makers (MMs, he), indexed by $n=1,2$, and one trader (she). We denote market maker $n$ by $\mathrm{MM} n$. At $t=1$, all market participants privately decide whether to observe $v$ for a cost $c>0$. Trade takes place at $t=2$ : the trader decides whether to submit a market order for one unit of the asset, and MMs simultaneously choose bid and ask prices. In the baseline model, the trader does not observe the prices before placing her market order; we relax this assumption in Section 6. There is price priority on the market, meaning that market orders are executed at the best possible price. The tie-breaking rule is specified as part of the equilibrium. The ask (respectively bid) price of MMn is denoted $a_{n}$ (resp. $b_{n}$ ). Hence, the trader's profit from a buy order (resp. sell order) is $v-\hat{a}($ resp. $\hat{b}-v)$, where $\hat{a}:=\min _{n} a_{n}$ and $\hat{b}:=\max _{n} b_{n}$; the profit of the MM executing the order is the opposite. For expository simplicity, we assume that MMs choose ask and bid prices in $[0,1]$.

We say that the trader is a speculator if her objective is to maximize her expected profit. The trader is a speculator with probability $\pi$, whereas with probability $1-\pi$ the trader is privately hit by a liquidity shock before her decision at $t=1$ : she then buys and sells the asset with equal probability independently of all other random variables of the model. In this case we say that the trader is a liquidity trader. To make the analysis interesting we assume $\pi \in(0,1)$. Figure 2 summarizes the timing of the model.

Equilibrium. Let $\mathrm{MM} n \mathrm{U}$ refer to $\mathrm{MM} n$ when he has not acquired information, and $\mathrm{MM} n \mathrm{H}$ (respectively $\mathrm{MM} n \mathrm{~L}$ ) refer to $\mathrm{MM} n$ when he has acquired information and observed $v=1$ 
(resp. $v=0$ ). A strategy of $\mathrm{MM} n$ comprises a probability $p_{n}$ of acquiring information at $t=1$, and cumulative distribution functions $\sigma_{n}, \underline{\sigma}_{n}$ and $\bar{\sigma}_{n}$ specifying respectively the distribution of the bid price $b_{n}$ of $\mathrm{MMn} \mathrm{U}, \mathrm{MM} n \mathrm{~L}$ and $\mathrm{MM} n \mathrm{H}$. As the bid and ask sides of the market are symmetric we assume, without loss of generality, that, conditional on $\mathrm{MM} n \mathrm{U}, 1-a_{n}$ is distributed like $b_{n}$. Similarly, we assume that $1-a_{n}$ conditional on $\mathrm{MM} n \mathrm{~L}$ (resp. $\mathrm{MM} n \mathrm{H}$ ) is distributed like $b_{n}$ conditional on $\mathrm{MM} n \mathrm{H}$ (resp. $\mathrm{MM} n \mathrm{~L}$ ). We will often use the index $m$ in conjunction with $n$, such that $n$ and $m$ represent the two MMs. A strategy of the speculator comprises a probability $q$ of acquiring information at $t=1$, as well as a market order as a function of her information at $t=2$. The equilibrium concept is perfect Bayesian equilibrium, with the tie-breaking rule specified as part of the equilibrium. ${ }^{7}$

The next definition will prove useful in the following sections. Roughly, a Wilson-EngelbrechtMilgrom-Weber-Lee (henceforth WELM) equilibrium is an equilibrium in which both MMs play the same strategy, and $\mathrm{MM} n \mathrm{~L}$ bids below $\mathrm{MM} n \mathrm{U}$ who himself bids below $\mathrm{MM} n \mathrm{H}{ }^{8}$

Definition 1. An equilibrium is a WELM equilibrium if it satisfies the following conditions:

(i) both MMs acquire information with probability $p$;

(ii) $M M s^{\prime}$ bidding strategies are identical: $\sigma_{1}=\sigma_{2}=\sigma, \underline{\sigma}_{1}=\underline{\sigma}_{2}=\underline{\sigma}$ and $\bar{\sigma}_{1}=\bar{\sigma}_{2}=\bar{\sigma}$;

(iii) $M M n L$ bids zero with probability 1: $\underline{\sigma}(0)=1$;

(iv) either $p \in\{0,1\}$ or $\sigma$ and $\bar{\sigma}$ are atomless, with supp $(\sigma)=[0, l]$ and supp $(\bar{\sigma})=[l, u]$.

We show below in Theorem 2 that any equilibrium of the baseline model is a WELM equilibrium. In Section 6 we discuss how WELM selects equilibria when quotes are observable.

\section{A One-Sided Strategic Complementarity}

In this section we fix the probabilities $p_{1}, p_{2}$ and $q$ with which MM1, MM2 and the speculator are informed and study the trading game that results. An equilibrium of this game will be referred to as a trading equilibrium. The trading game is formally equivalent in the case $q=0$ to a first-price sealed-bid common-value auction with (possibly) asymmetrically informed bidders. ${ }^{9}$ In contrast to that literature however, our objective is to study and

\footnotetext{
${ }^{7} \mathrm{~A}$ tie-breaking rule specifies the probabilities, in case of ties, with which market orders are executed by MM1, as a function of the MMs' quotes.

${ }^{8}$ After Wilson (1967), Engelbrecht-Wiggans, Milgrom and Weber (1983), and Lee (1984).

${ }^{9}$ Such auctions have been analyzed, as already mentioned, by Wilson (1967), Engelbrecht-Wiggans et al. (1983), and Lee (1984).
} 
compare profits on the two sides of a dealer market, and to analyze the effect of information acquired by one side of the market on incentives to acquire information on the other side. All proofs of this section are in Appendix A.

As the equilibrium structure of the trading game closely resembles that found in the aforementioned literature, the formal definitions and detailed analysis are relegated to Online Appendix C. We briefly summarize below the main ideas and then move on to analyzing how the information probabilities $p_{1}, p_{2}$ and $q$ affect profits in the trading game.

In the trading game, the MMs and the trader simultaneously post, respectively, quotes and a market order. Except in knife-edge cases, in any trading equilibrium the speculator sells (respectively buys) with probability 1 when she is informed and observed $v=0$ (resp. $v=1$ ), and abstains when she is uninformed. For the informed speculator the intuition is straightforward, since both bid and ask prices are in the unit interval. Since MMs set quotes so as to not lose money on average, the uninformed speculator cannot make positive expected profit from trading, and optimally abstains. The support of the equilibrium bid distributions of different types of a given MM never strictly overlap: $\mathrm{MM} n \mathrm{~L}$ bids below $\mathrm{MM} n \mathrm{U}$, who himself bids below MMnH. ${ }^{10}$ To see why, fix the bid of $\mathrm{MM} n \mathrm{~L}$ at zero and suppose for the sake of argument that we can find $0<b^{\prime}<b^{\prime \prime}$ such that MMnU and MMnH both bid at $b^{\prime}$ and at $b^{\prime \prime}$ with positive probability. In this case MMnH must be indifferent between the two. Thus, conditional on $V=1, \mathrm{MM} n \mathrm{U}$ is also indifferent between $b^{\prime}$ and $b^{\prime \prime}$. However, conditional on $V=0, \mathrm{MM} n \mathrm{U}$ strictly prefers $b^{\prime}$ to $b^{\prime \prime}$, since bidding $b^{\prime}$ gives a smaller winning probability, and a smaller loss in case of winning. Hence, $\mathrm{MM} n \mathrm{U}$ strictly prefers $b^{\prime}$ over $b^{\prime \prime}$, contradicting the hypothesis that $\mathrm{MMnU}$ chooses both with positive probability.

We now analyze profits. We define an informed market participant's expected trading profit as his/her expected profit in the trading game when he/she is informed, and similarly for an uninformed market participant. ${ }^{11}$ Trading profits are gross profits, that is, profits obtained before subtracting the cost of information. We show the following result.

Lemma 1. For all $p_{1}, p_{2}$ and $q$, a trading equilibrium exists. Any given market participant's expected trading profit when informed is independent of the trading equilibrium considered. Similarly, any given market participant's expected trading profit when uninformed is independent of the trading equilibrium considered.

\footnotetext{
${ }^{10} \mathrm{MMnL}$ always bids zero. MMnU never bids above $\frac{1}{2}$. Both $\mathrm{MMnU}$ and $\mathrm{MMnH}$ use mixed bidding strategies unless either $p_{1}=p_{2}=0$ or $p_{1}=p_{2}=1$.

${ }^{11}$ For definiteness, in the case of an informed market participant the expectation is calculated before observing $v$. Of course, in equilibrium, the symmetry of the bid and ask sides of the market makes this distinction irrelevant: expected profits before and after observing $v$ are then the same thing.
} 
We now let $\Pi_{n}$ (respectively $\bar{\Pi}_{n}$ ) denote the equilibrium expected trading profit of MMn when uninformed (resp. informed), and $\Pi_{S}$ (respectively $\bar{\Pi}_{S}$ ) denote the equilibrium expected trading profit of the speculator when uninformed (resp. informed).

Lemma 2. Suppose $p_{n}>p_{m}$. Then $\bar{\Pi}_{m}=\bar{\Pi}_{n}>\Pi_{n}>\Pi_{m}=0$.

In equilibrium, both MMs earn the same (expected) profit when informed. Intuitively, if $\mathrm{MM} n \mathrm{H}$ were to make more profit than $\mathrm{MM} m \mathrm{H}$, then $\mathrm{MM} m \mathrm{H}$ could increase his profit by bidding just above MMnH's highest bid price. Next, if $p_{n}>p_{m}$ then $\mathrm{MM} n \mathrm{U}$ makes greater profit than $\mathrm{MM} m \mathrm{U}$. The logic is straightforward: informed MMs pick a disproportionate share of profitable market orders; since $\mathrm{MM} n$ is more often informed than $\mathrm{MM} m, \mathrm{MM} m \mathrm{U}$ then faces more adverse selection than $\mathrm{MM} n \mathrm{U}$. Intuitively, an uninformed MM extracts rent from a competitor's belief that he is informed with high probability. For our purpose the main implication is the following: $p_{n}>p_{m}$ implies $\bar{\Pi}_{n}-\Pi_{n}<\bar{\Pi}_{m}-\Pi_{m}$, that is, MMm's incremental profit from being informed is greater than that of $\mathrm{MM} n$. Consequently, any equilibrium must be such that $p_{1}=p_{2}$ (in fact, any equilibrium of the baseline model is a WELM equilibrium; see Theorem 2). We therefore focus in the rest of the section on profiles of information acquisition satisfying $p_{1}=p_{2}$, and let $p$ denote the common probability with which MMs are informed. The next theorem is the central result of this section.

Theorem 1. There is a one-sided strategic complementarity in information acquisition.

1. The more information acquired by any market participant the smaller the speculator's gain from acquiring information: $\bar{\Pi}_{S}-\Pi_{S}$ is decreasing in $p$ and $q$.

2. By contrast, information acquired by the speculator enhances $M M s^{\prime}$ gain from acquiring information: $\bar{\Pi}_{n}-\Pi_{n}$ is decreasing in $p$ but increasing in $q$.

The logic behind the effects of $p$ and $q$ on the speculator's gain from being informed is as follows. The speculator's only chance of making a profit is against uninformed MMs. Raising $p$ therefore reduces the speculator's chances of finding a profitable trade. ${ }^{12}$ Higher $q$, on the other hand, generates greater adverse selection for uninformed MMs, which in turn induces wider spreads. Increasing $q$ thus reduces the profit made by the speculator on each profitable trade.

\footnotetext{
${ }^{12}$ Moreover, raising $p$ increases adverse selection for uninformed MMs which, in turn, induces the latter to set wider bid-ask spreads, further reducing the informed speculator's expected trading profit.
} 
The effect of $p$ on MMs' gain from information is straightforward, as higher $p$ means greater competition for profitable market orders. The effect of $q$ on MMs' gain from information is the more interesting effect. Increasing $q$ worsens the adverse selection problem faced by all uninformed MMs, which induces them to set wider spreads. This, in turn, softens price competition for informed MMs, who now increase their own spreads. MMs' incremental trading profit from being informed therefore increases in the probability with which the speculator acquires information.

\section{Information Acquisition}

In this section we analyze equilibrium information acquisition in the baseline model. We show that the pattern of information acquisition is uniquely determined as a function of information cost and market composition. In particular, at small information costs MMs acquire information whereas the speculator chooses to remain uninformed. The situation is reversed at larger costs. The next theorem is this section's central result. All proofs of this section are in Appendix A.

Theorem 2. There exists an equilibrium. Moreover, any equilibrium is a WELM equilibrium. The information acquisition probabilities, $p$ and $q$, are independent of the equilibrium considered; $p$ is non-increasing in $c$ and tends to 1 as $c$ tends to 0 . For $c>1 / 2$, neither the speculator nor the MMs acquire information, and there exist $0<\underline{c}<\bar{c}<\frac{1}{2}$ such that:

- if $c \in(0, \underline{c})$ then $M M s$ acquire information with positive probability but the speculator remains uninformed;

- if $c \in(\underline{c}, \bar{c})$ then the $M M s$ and the speculator all acquire information with positive probability;

- if $c \in\left(\bar{c}, \frac{1}{2}\right)$ then the speculator acquires information with positive probability but MMs remain uninformed.

In view of Theorem 2, the standard assumptions about informational asymmetries in dealer markets whereby informed speculators and liquidity traders trade with uninformed MMs are warranted for a range of high information costs $\left(c \in\left(\bar{c}, \frac{1}{2}\right)\right)$. Yet, strikingly, at lower costs $(c \in(0, \underline{c}))$ the market consists only of liquidity traders, informed MMs and uninformed MMs. The equilibrium pattern of information acquisition is illustrated in Figure 3, panel A, for 


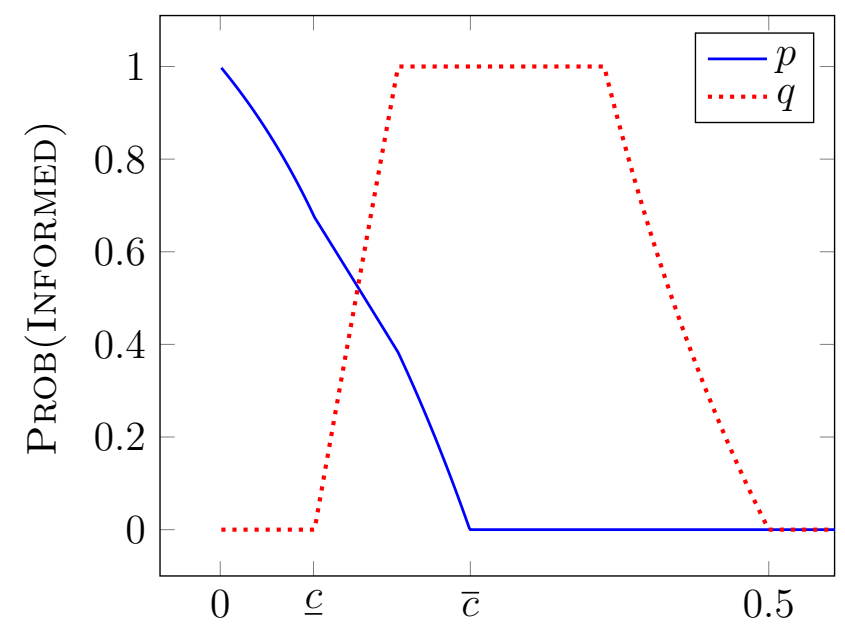

(A) Information Cost, $c$

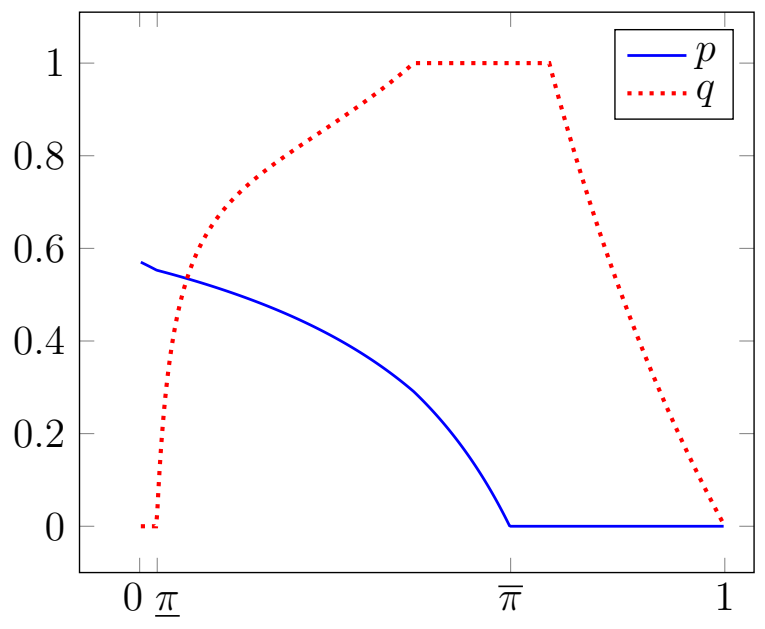

(B) Fraction of Speculators, $\pi$

Figure 3: Equilibrium Information Acquisition

$\pi=\frac{3}{10} .{ }^{13}$ The information acquisition probabilities are on the vertical axis; the information cost is on the horizontal axis. The solid curve shows the equilibrium $p$, and the dotted curve the equilibrium $q$. We also indicate the cutoff $\underline{c}>0$ below which the speculator is uninformed, and the cutoff $\bar{c}<\frac{1}{2}$ above which MMs are uninformed.

The uniqueness of $p$ and $q$ as well the non-increasingness of $p$ as a function of $c$ all follow from Theorem 1. We summarize in the next paragraphs the logic behind who acquires information. Notice that in a WELM equilibrium uninformed market participants make zero expected profits $\left(\Pi_{n}=\Pi_{S}=0\right)$ so gains from being informed equal expected profits when informed.

At small information costs, MMs acquire information. As uninformed MMs never set bid prices above $\frac{1}{2}$ nor set ask prices below $\frac{1}{2}, \mathrm{MM} n$ 's gain from being informed in the trading game is at least as large as $\frac{(1-p)(1-\pi)}{4}$ : there is probability $(1-p)$ that $\mathrm{MM} m$ is uninformed, in which case the informed type of $\mathrm{MM} n$ ensures profit $\frac{1}{2}$ whenever the trader is a liquidity trader who either sells when $V=1$ or buys when $V=0$, which occurs with probability $\frac{1-\pi}{2}$. Thus, in equilibrium, $\frac{(1-p)(1-\pi)}{4} \leq c$, and $p$ tends to 1 as $c$ tends to $0 .{ }^{14}$

\footnotetext{
${ }^{13}$ The code used for calculating the equilibrium and simulating the prices in the following figures is available on the authors' websites.

${ }^{14}$ If $\frac{(1-p)(1-\pi)}{4}>c$ then a MM's gain from acquiring information is larger than the cost of information, implying $p=1$. But this is a contradiction, since $c>0$.
} 
At small information costs, MMs crowd out the speculator. By symmetry of the bid and ask sides of the market, the speculator's gain from being informed in the trading game may be written as

$$
\bar{\Pi}_{S}-\Pi_{S}=\left(1-p^{2}\right) \mathbb{E}[\hat{b} \mid V=0, \text { either } 1 \text { or } 2 \text { MMs are uninformed }]
$$

The factor $1-p^{2}$ represents the speculator's chances of making a positive profit, which will only occur if at least one MM is uninformed. The second term is the expectation of the best bid price, conditional on $V=0$ and at least one MM being uninformed. We established earlier that $\frac{(1-p)(1-\pi)}{4} \leq c$, thus,

$$
1-p^{2} \leq \frac{4 c(1+p)}{1-\pi} \leq \frac{8 c}{1-\pi}
$$

Consider now the expectation of the best bid price appearing on the right-hand side of (1). As $p$ tends to 1, an uninformed MM is almost exclusively left with unprofitable market orders (when $p$ is close to 1 an uninformed MM expects profitable market orders to be picked off by his competitor). Since $p$ tends to 1 when $c$ tends to 0 , an uninformed MM's bid-ask spread therefore tends to 1 as $c$ tends to 0 , giving ${ }^{15}$

$$
\lim _{c \rightarrow 0} \mathbb{E}[\hat{b} \mid V=0, \text { either } 1 \text { or } 2 \text { MMs are uninformed }]=0 .
$$

Lastly, combining (1), (2) and (3) gives $\bar{\Pi}_{S}-\Pi_{S}<c$ for all $c$ sufficiently small. This implies that the speculator chooses to remain uninformed at small information costs.

At high information costs, the speculator acquires information whereas MMs remain uninformed. Fix $p=0$ and $q=1$, and consider the resulting trading game. With both MMs uninformed, Bertrand competition yields $\hat{b}=\mathbb{E}[V \mid$ sell $]=\frac{1-\pi}{2}=1-\hat{a} \cdot{ }^{16}$ The informed speculator sells when $V=0$ and buys when $V=1$. Hence,

$$
\bar{\Pi}_{S}-\Pi_{S}=\frac{1}{2} \hat{b}+\frac{1}{2}(1-\hat{a})=\frac{1-\pi}{2} .
$$

Now, if a MM were informed he would pick all profitable market orders, that is, all sell orders of the liquidity trader when $V=1$ and all buy orders of the liquidity trader when $V=0$. We

\footnotetext{
${ }^{15} \mathrm{~A}$ formal proof of (3) is provided in Appendix A.

${ }^{16}$ As $q=1$, the speculator sells when $V=0$ and buys when $V=1: \mathbb{E}[V \mid$ sell $]=\frac{\frac{1}{2} \cdot \frac{1-\pi}{2}}{\frac{1}{2} \cdot \frac{1-\pi}{2}+\frac{1}{2}\left(\frac{1-\pi}{2}+\pi\right)}=\frac{1-\pi}{2}$.
} 
therefore obtain

$$
\bar{\Pi}_{n}-\Pi_{n}=(1-\pi)\left(\frac{1}{4}(1-\hat{b})+\frac{1}{4} \hat{a}\right)=\frac{1-\pi^{2}}{4} .
$$

Combining (4) and (5) yields $\bar{\Pi}_{n}-\Pi_{n}<\bar{\Pi}_{S}-\Pi_{S}$. The latter inequality establishes that, for $c \in\left[\frac{1-\pi^{2}}{4}, \frac{1-\pi}{2}\right]$, the unique equilibrium information acquisition probabilities are $q=1$ and $p=0$ : the speculator acquires information whereas MMs remain uninformed. Intuitively, MMs face execution risk, which the speculator does not. Hence if spreads are not too wide, such that MMs and speculator face not too dissimilar prices, then the speculator's gain from becoming informed is larger than the corresponding gain of MMs.

We end this section by investigating the effect of the composition of the market (in terms of speculation versus liquidity trading) on information acquisition.

Proposition 1. Assume $c<\frac{1}{2}$. There exist $0 \leq \underline{\pi} \leq \bar{\pi}<1$ such that, in any equilibrium:

- if $\pi \in(0, \underline{\pi})$ then MMs acquire information with positive probability but the speculator remains uninformed;

- if $\pi \in(\underline{\pi}, \bar{\pi})$ then the $M M s$ and the speculator all acquire information with positive probability;

- if $\pi \in(\bar{\pi}, 1)$ then the speculator acquires information with positive probability but MMs remain uninformed.

Moreover, there exists $c^{*}>0$ such that $\underline{\pi}>0$ if and only if $c<c^{*}$. Lastly, the equilibrium probability $p$ with which a MM acquires information is non-increasing in $\pi$, and the equilibrium probability $q$ with which the speculator acquires information tends to 0 as $\pi$ tends to 1.

The proposition is illustrated in Figure 3, panel B, for $c=0.15$. The vertical axis shows information acquisition, with $\pi$ on the horizontal axis. The MMs exclusively recoup $c$ by executing market orders from the liquidity trader. So MMs acquire information when liquidity trades are frequent and remain uninformed when liquidity trades are rare. The speculator, on the other hand, exclusively recoups $c$ by trading with uninformed MMs. Therefore, the speculator remains uninformed when liquidity trades are frequent, in which case most MMs are informed, and acquires information when liquidity trades are rare, in which case most MMs are uninformed. The speculator's information acquisition probability tends to 0 as $\pi$ tends to 1 . If this were not the case then, conditional on $V=0, \hat{b}$ would have to converge to 


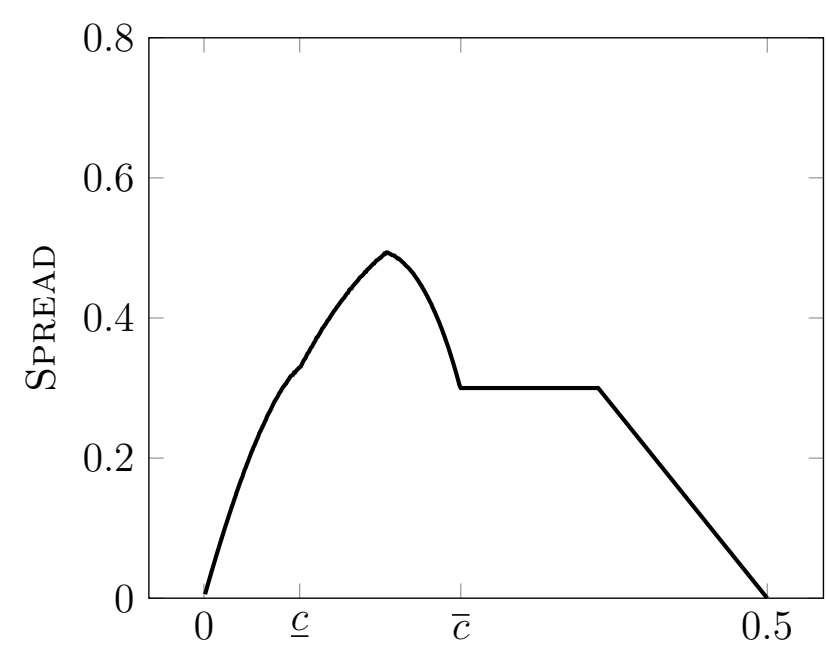

(A) Information Cost, $c$

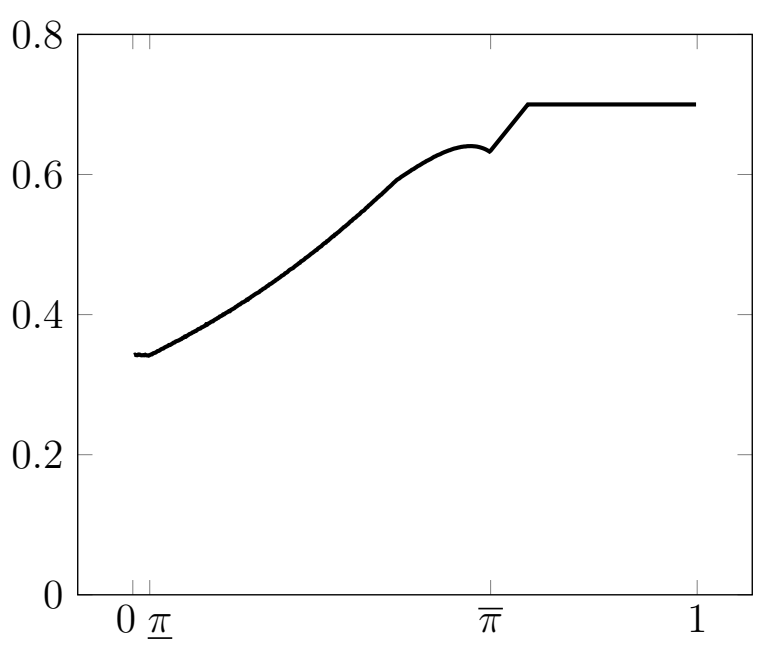

(B) Fraction of Speculators, $\pi$

Figure 4: Market Liquidity

0 (in probability) since the probability of a market order coming from the speculator would tend to 1 as $\pi$ tends to 1 . Similarly, conditional on $V=1, \hat{a}$ would have to converge to 1 . However, in that case, the speculator would have no incentive at all to pay for information.

\section{$5 \quad$ Market Liquidity and Price Discovery}

The model's implications for the spread (measuring "inverse" market liquidity) and squared price error (measuring "inverse" price discovery) contrast with the predictions of models in which MMs are uninformed. In a model where only speculators can acquire information, increasing the cost of information would reduce informed trading and, thereby, adverse selection too. The combination of these effects would in turn decrease the spread and increase the squared price error. By contrast, in our model increasing $c$ can increase the expected spread and reduce the expected squared price error (thus reducing market liquidity and improving price discovery). We also show that increasing $\pi$ can reduce the expected spread and increase the expected squared price error (thus improving market liquidity and reducing price discovery). Below we use "spread" and "squared price error" to refer to these variables' expected values. ${ }^{17}$ We next discuss these implications in turn.

\footnotetext{
${ }^{17}$ Specifically, throughout this section the spread refers to $s:=\mathbb{E}[\hat{a}-\hat{b}]$. The squared price error refers to $d:=\mathbb{E}\left[(r-V)^{2}\right]$, with $r$ denoting the realized price: $r=\hat{a}$ in case of a buy order, $r=\hat{b}$ in case of a sell order, and $r=\frac{\hat{a}+\hat{b}}{2}$ if the trader abstains.
} 


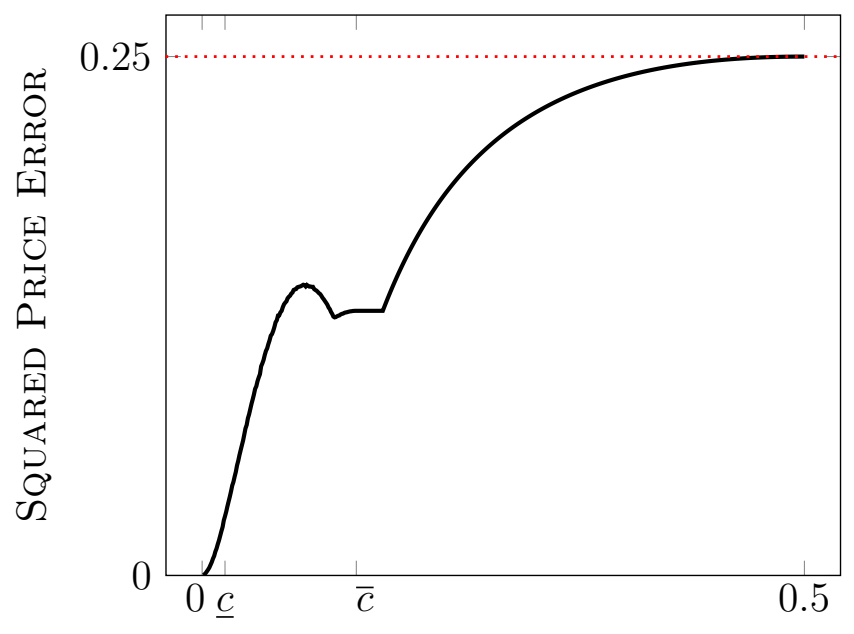

(a) Information Cost, $c$

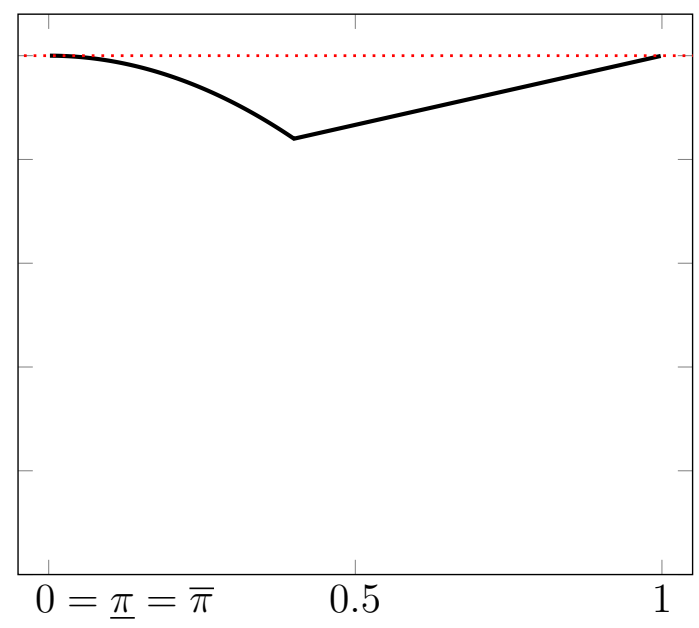

(B) Fraction of Speculators, $\pi$

Figure 5: Price Discovery

Increasing the cost of information can increase the spread. The mechanism is as follows. Suppose $v=1$, to fix ideas. Each MM acquires information with probability converging to 1 as $c$ tends to 0 (Theorem 2). Informed MMs competing to offer the best bid price then ensure that $\hat{b}$ converges (in probability) to 1 as $c$ tends to 0 . Hence, as $v=1$ implies $\hat{a}=1$ whenever both MMs are informed, the spread converges (in probability) to 0 as $c$ tends to 0 . But then, starting at a very small $c$, increasing the cost must increase the spread. We illustrate this effect in Figure 4, panel A, for $\pi=\frac{3}{10}$.

Increasing the cost of information can reduce the squared price error. The reason is that, in an intermediate cost range, raising $c$ induces the speculator to acquire more information (Theorem 2; see also Figure 3, panel A). In this range, raising $c$ in addition leads MMs to acquire less information. However, due to the one-sided strategic complementarity highlighted in Theorem 1, in this cost range $q$ rises faster than $p$ falls: so the negative impact on the squared price error resulting from the speculator's increased information acquisition dominates the positive impact resulting from MMs' reduced information acquisition. The resulting effect is illustrated in Figure 5, panel A, for $\pi=\frac{7}{10}$.

Increasing the fraction of speculators in the market can reduce the spread. Immediately below $\pi=\bar{\pi}$, the probability $q$ with which the speculator acquires information is invariant in $\pi$, yet $p$ decreases with $\pi$ (see Figure 3, panel B). This in turn reduces uninformed 
MMs' adverse selection problem, who therefore narrow their spreads. We illustrate this effect in Figure 4, panel B, for $c=0.15$.

\section{Increasing the fraction of speculators in the market can increase the squared price}

error. This finding is a consequence of the market collapse resulting from excessively scarce liquidity trading: a high fraction of speculators induces very large spreads which, in turn, dissuades speculators from acquiring information (and, therefore, trading). Increasing the fraction of speculators can thus reduce speculators' market participation and via this channel increase the squared price error. We illustrate this effect in Figure 5, Panel B, for $c=\frac{3}{10}$.

\section{Observable Quotes}

In this section we extend the baseline model of Section 2 by letting the MMs' quotes be observable with probability $z$ before the trader's decision in period $t=2$; the baseline model corresponds to $z=0 .{ }^{18}$ The timing of the observable quotes model is illustrated in Figure 6. The trader is privately hit by the liquidity shock with probability $1-\pi$, after which all market participants privately decide whether to observe $v$, for a cost $c>0$. The MMs then simultaneously choose bid and ask prices. ${ }^{19}$ The trader observes the quotes with probability $z$; she then either abstains or places a market order for one unit of the asset. All proofs of this section are in Appendix B.

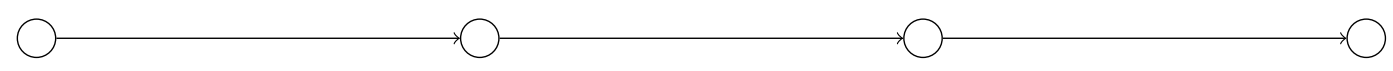

$\begin{array}{cccc}\text { Liquidity shock with } & \text { Covert information } & \text { MMs simultaneously } & \text { Trader observes prices } \\ \text { probability } 1-\pi & \text { acquisition by MMs } & \text { set prices } & \text { with probability } z \text { and } \\ & \text { and speculator } & & \text { submits market order }\end{array}$

Figure 6: Timing - Observable Quotes

\footnotetext{
${ }^{18}$ The cases $z=0$ and $z=1$ are arguably the cases with the most applied interest; for completeness and in order to derive further comparative statics, we allow however $z$ to take any value in $[0,1]$.

${ }^{19}$ We assume for tractability that the MMs set prices simultaneously, but note that in many modern markets MMs would also be able to observe each others' quotes.
} 
Relative to the baseline model, making the quotes observable adds several layers of complexity:

- Each MM's decision to acquire information now induces additional externalities on the other MM: information which the speculator learns through the prices of one MM may be used to make profit against the other MM. Two interesting consequences of this feature are that:

(i) MM's gain from acquiring information is increasing in quote observability, $z$;

(ii) MM's gain from acquiring information can increase with MMs' information, $p$.

- As the speculator can now learn about $v$ without acquiring information herself, observable quotes reduce the speculator's gain from acquiring information.

- Observable quotes allow MMs to manipulate demand through prices. For instance, $\mathrm{MM} n \mathrm{U}$ could masquerade as $\mathrm{MM} n \mathrm{H}$ and reduce adverse selection by "jamming" the signal of MMmL.

To make progress and keep the analysis tractable we restrict attention throughout this section to WELM equilibria. ${ }^{20}$ While multiple WELM equilibria exist, we show in Online Appendix D that all WELM equilibria share important common properties. First, as MMs (optimally) reveal their information to the trader, the speculator who does not acquire information learns $v$ with probability $z\left[1-(1-p)^{2}\right]$, that is, as long as the speculator gets to observe the quotes and at least one MM is informed. The speculator trades if she acquires information directly, or if she learns $v$ indirectly through a MM's quotes; she abstains otherwise. Second, given $p$ and $q$, a market participant's expected trading profit when he/she acquires information is the same in all WELM trading equilibria. ${ }^{21}$ Similarly, his/her expected trading profit when he/she does not acquire information is the same in all WELM trading equilibria. We thus extend previous notation and let $\Pi_{n}$ (respectively $\bar{\Pi}_{n}$ ) denote MMn's equilibrium expected trading profit when he does not acquire information (resp. when he does acquire

\footnotetext{
${ }^{20}$ Theorem 2 ruled out non-WELM equilibria in the case $z=0$. In WELM equilibria different MM types bid in non-overlapping intervals; so when quotes are observable, an informed MM (optimally) reveals his information to the trader. Whether non-WELM equilibria exist for $z>0$ remains an open question.

${ }^{21}$ We use the terminology WELM trading equilibrium for a perfect Bayesian equilibrium of the trading game induced by the observable quotes model that satisfies: (i) $\sigma_{1}=\sigma_{2}=\sigma, \underline{\sigma}_{1}=\underline{\sigma}_{2}=\underline{\sigma}$ and $\bar{\sigma}_{1}=\bar{\sigma}_{2}=\bar{\sigma}$; (ii) $\underline{\sigma}(0)=1$; (iii) either $p \in\{0,1\}$ or $\sigma$ and $\bar{\sigma}$ are atomless, with supp $(\sigma)=[0, l]$ and $\operatorname{supp}(\bar{\sigma})=[l, u]$. See Online Appendix D.
} 
information), and $\Pi_{S}$ (respectively $\bar{\Pi}_{S}$ ) denote the speculator's equilibrium expected trading profit when she does not acquire information (resp. when she does acquire information).

We explore first the effect of the observability of the quotes on market participants' gains from acquiring information.

Proposition 2. The greater the observability of the quotes the smaller the speculator's gain from acquiring information: if $p>0$ and $q<1$ then $\bar{\Pi}_{S}-\Pi_{S}$ is decreasing in $z$. By contrast, the observability of the quotes enhances $M M s^{\prime}$ gain from acquiring information: if $p>0$ and $q<1$ then $\bar{\Pi}_{n}-\Pi_{n}$ is increasing in $z$.

The first part of the proposition is straightforward. As long as (i) the speculator does not acquire information with probability 1 and (ii) MMs acquire information with positive probability then, increasing $z$ : (a) increases the amount of information which the speculator can retrieve from the quotes, (b) induces MMs to set wider spreads, by exposing uninformed MMs to greater adverse selection. Both effects in turn decrease the speculator's incentive to pay for information. The second part of the proposition is more interesting. As noted above, increasing $z$ exposes uninformed MMs to greater adverse selection, inducing them to set wider spreads. The larger spreads of the uninformed MMs enable in turn informed MMs to turn a greater profit from trading with the liquidity trader. ${ }^{22}$

Our next result extends Theorem 1.

Theorem 3. There is a one-sided strategic complementarity in information acquisition and, for $\pi$ and $z$ sufficiently large, also a strategic complementarity in information acquisition amongst $M M s^{23}$

1. The more information acquired by any market participant the smaller the speculator's gain from acquiring information: $\bar{\Pi}_{S}-\Pi_{S}$ is decreasing in $p$ and $q$.

2. By contrast: $\bar{\Pi}_{n}-\Pi_{n}$ is increasing in $q$ and, for $\pi$ and $z$ sufficiently large, can be either increasing or decreasing in $p$.

The logic underlying the one-side strategic complementarity in information acquisition is as in the baseline model. ${ }^{24}$ The new result relative to Theorem 1 is the impact of $p$ on MMs'

\footnotetext{
${ }^{22}$ This mechanism is naturally akin to the mechanism in Theorem 1 that induced MMs' gain from information to increase with $q$ : increasing $z$ indirectly increases information available to the speculator.

${ }^{23}$ The results hold for all $\pi$ and $z$, except where explicitly stated.

${ }^{24}$ Note however that with $z>0$, the adverse effect of $p$ on the speculator's incentive to acquire information is even larger than before since now increasing $p$ channels information concerning $v$ to the speculator.
} 
gain from acquiring information. On one hand, increasing $p$ enhances competition among MMs for profitable market orders; this effect reduces MMs' gain from acquiring information, and led $\bar{\Pi}_{n}-\Pi_{n}$ to be decreasing in $p$ at $z=0$. On the other hand, with observable quotes, increasing $p$ channels additional information to the speculator, exposing uninformed MMs to greater adverse selection. The larger spreads of the uninformed MMs enable in turn informed MMs to make greater profits from trading with the liquidity trader. This feedback effect implies that, if $\pi$ and $z$ are sufficiently large, then MMs' gain from acquiring information increases with $p$. Hence, the mechanism at play in the one-sided strategic complementarity in information induces, when quotes are observable, a strategic complementarity in information acquisition amongst MMs.

The feedback effect highlighted above opens the door to the potential existence of multiple WELM equilibria, but the model's main predictions continue to hold. Who acquires information crucially depends on the cost of information: when this cost is small, MMs acquire information and crowd out the speculator; by contrast, when this cost is large, the speculator acquires information and MMs remain uninformed.

Theorem 4. For any $z$, there exist $0<\underline{c}<\bar{c}<\frac{1}{2}$ such that, for $c \in(0, \underline{c}) \cup\left(\bar{c}, \frac{1}{2}\right)$, a WELM equilibrium exists and in any WELM equilibrium:

- if $c \in(0, \underline{c})$ then $M M s$ acquire information with positive probability but the speculator remains uninformed;

- if $c \in\left(\bar{c}, \frac{1}{2}\right)$ then the speculator acquires information with positive probability but MMs remain uninformed.

Figure 7 illustrates Theorem 4, for $\pi=\frac{3}{10}$. The dashed (respectively dotted) curve corresponds to equilibrium $p$ (resp. q) values in the baseline model, that is, $z=0$; the solid (respectively dash-dotted) curve corresponds to equilibrium $p$ (resp. $q$ ) values in the model with perfectly observable quotes, that is, $z=1$. First, notice that $q$ is weakly lower for $z=1$ than for $z=0$, capturing the fact that observable quotes reduce the speculator's incentive to pay for information (Proposition 2). By contrast the cutoff $\bar{c}$ above which MMs are uninformed does not depend on $z$. To see this, note that if $p=0$ is an equilibrium outcome for $z=0$ then $p=0$ is also an equilibrium outcome for $z=1$ : intuitively, if MMs are uninformed then quote observability has no effect on any of the market participants' profits. Next, going from left to right in the figure, observe that whereas MMs initially acquire more information with observable quotes than without, things eventually reverse within the cost interval where 


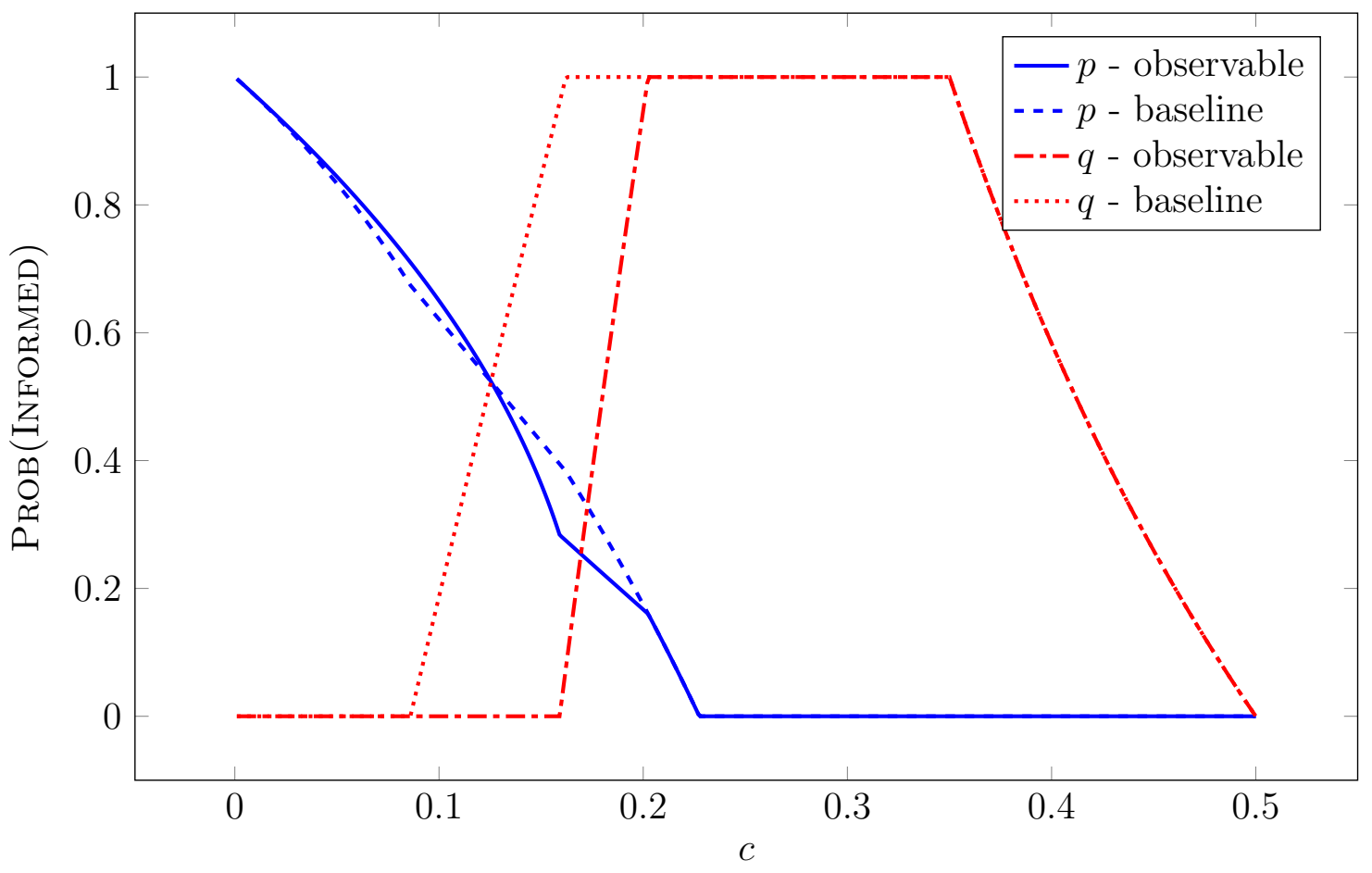

Figure 7: Equilibrium Information Acquisition:

Baseline vs. Observable Quotes

$q$ is greater for $z=0$ than for $z=1$. The logic is the following. We saw in Proposition 2 that, fixing $p$ and $q$, MMs' gain from acquiring information increases with $z$. Yet we also established (Theorem 3) that MMs' gain from acquiring information increases with $q$. In the cost interval where increasing $z$ reduces $q$, the reduction in $q$ is eventually sufficiently large that increasing $z$ reduces $p$. Finally, notice that when $q=1 \mathrm{MMs}$ ' information acquisition is the same in the baseline and observable quotes models since, being already informed, the speculator then learns nothing from the quotes.

\section{Concluding Remarks}

This paper analyzes information acquisition in dealer markets. We identify a one-sided strategic complementarity in information acquisition: the more informed traders are, the larger MMs' gain from becoming informed. We also identify a strategic complementarity in information acquisition amongst MMs, that arises when quotes are observable. The paper's main result shows that the microstructure of dealer markets pins down information acquisition. 
The pattern often assumed in the literature wherein traders are superiorly informed relative to MMs arises at high costs. But other patterns arise at different costs. In particular, at small costs, information acquisition is reversed. In that case, MMs are informed, but traders are not. Furthermore, when most traders are speculators, the speculators choose to acquire information whereas MMs remain uninformed; by contrast, when most traders are liquidity traders, MMs acquire information and traders remain uninformed. The model has striking implications for market liquidity and price discovery: decreasing the cost of information and increasing the share of speculators can improve liquidity; increasing the cost of information and decreasing the fraction of speculators can improve price discovery.

Discussion of assumptions. The model we propose is stylized, yet rich enough to deliver various seemingly robust insights concerning the implications of the microstructure of dealer markets on incentives to invest in information. The baseline model (Section 2) illustrates these insights in the simplest possible way. The observable quotes model (Section 6) opens a new information flow from MMs to traders, since traders benefit from MMs' information acquisition via more informative quotes. The fundamental mechanisms, however, are the same in the two models. Loosely speaking, increasing $p$ in the observable quotes model is like increasing $p$ and $q$ in the baseline model.

Some of our modelling assumptions are relatively easily relaxed, including the number of MMs, the probability that $V=1$, or even the binary nature of the asset value, $V$. But two assumptions appear necessary for the tractability of the model:

(i) There is a single trading round. Introducing multiple trading rounds would imply that private information leaks to the market through previous quotes and trades, and that the same information can be used in several trading rounds. In this case, on the one hand MMs and traders have an incentive to act strategically and hide their private information so as to use it several times by setting less aggressive quotes or trading less aggressively, but on the other hand, they also have an incentive to act quickly before the information of other market participants is disseminated to the market. The reaction to these incentives will in turn determine how 'many times' information can be used, and how profitable it will be, thus modifying the incentives to acquire information.

(ii) The market participants acquire perfect information concerning $V$. Of course, in practice traders and MMs can incur varying costs in order to acquire more or less accurate information about the assets they trade. One could as a first step improve the realism 
of the baseline model (i.e., with unobservable quotes) by supposing that, instead of observing the realization of $V$, market participants can, for a cost $c>0$, observe the realization of a binary signal correlated with $V$. However, noisy signals of $V$ imply that informed MMs also learn from the order flow.

Relation to empirics. Our findings shed light on several well-documented empirical regularities. First, both traders and MMs may have proprietary information. Manaster and Mann (1996), for instance, provide evidence in connection with the market for commodity futures, Li and Heidle (2004) for stockmarkets, and Covrig and Melvin (2002) and Sapp (2002) for the foreign exchange market. Therefore, traders cannot be viewed purely as uninformed liquidity traders, and MMs cannot be viewed as only learning from their private knowledge of the order flow. ${ }^{25}$

Second, dealer-driven price discovery can be more important than trader-driven price discovery. For stock markets, Anand and Subrahmanyam (2006) find that “... intermediaries appear to be more informed than all other institutions and individuals combined." Valseth (2013) explores government bond markets and compares the informational content of the interdealer and customer order flows: the interdealer order flow explains almost a quarter of daily yield variation, whereas the customer order flow has little explanatory power.

Third, MMs are often asymmetrically informed. The finding is widely documented (Albanesi and Rindi, 2000; Huang, 2002; Massa and Simonov, 2003). In our setting, ex ante identical MMs play mixed information acquisition strategies in equilibrium, and may therefore be ex-post asymmetrically informed.

Fourth, more volatile assets exhibit larger spreads. Stoll (1978) was first to provide evidence in the case of stocks, while Chen, Lesmond and Wei (2007) find that spreads are higher for corporate bonds with lower rating or higher maturity, which are both associated with higher price volatility. ${ }^{26}$ We establish that the bid-ask spread is largest when there is both informed market making and informed trading. Consequently, in our setting, spreads are maximized when prices are volatile. ${ }^{27}$

\footnotetext{
${ }^{25} \mathrm{MMs}$ acquiring information through this channel have been considered in, for instance, Leach and Madhavan (1993), Bloomfield and O'Hara (2000) and de Frutos and Manzano (2005). In this literature, the focus is on MMs' incentives to experiment with prices in order to learn new information.

${ }^{26}$ Edwards, Harris and Piwowar (2007) and Bao, Pan and Wang (2011) find similar evidence, but their measure of market liquidity is different.

${ }^{27}$ When MMs randomize between acquiring information and not, each MM is uncertain about the information of her competitors. The trading equilibrium thus involves mixing on the part of all MMs.
} 
Dealer markets, electronic limit order markets and high-frequency trading. In the spirit of Glosten and Milgrom (1985), our model is that of a classic dealer market, with MMs supplying liquidity on one side of the market and traders consuming liquidity on the other. Dealer markets comprise some of the world's largest financial markets, including foreign exchange (FX), corporate bond and swap markets (see, e.g., Hasbrouck (2007)). In stock markets, traditional designated market makers (NYSE specialists, Nasdaq MMs) have largely been displaced, and replaced by "voluntary", or "de facto", market makers. ${ }^{28}$

More generally, the advent of electronic exchanges has facilitated a rise in the number of independent firms and investors supplying liquidity by posting limit orders, and a central feature of the rapidly growing literature on limit order markets is precisely the choice between liquidity provision and liquidity consumption (e.g. Foucault (1999)). Yet, even though electronic limit order markets allow traders to choose between demanding and supplying liquidity, high-frequency traders (HFTs) have a natural advantage in liquidity supply. Limit orders run the risk of being adversely picked off if the security's value moves past the limit price and the limit order is hit before it can be cancelled. HFTs can update limit orders fast and are therefore less exposed to adverse selection than ordinary traders, who are, in effect, driven out of market-making activities. Quoting Menkveld (2016), "A formal definition does not exist but most associate HFT with extremely fast computers running algorithms coded by traders who trade for their own account. ... These traders typically do not work at the deep-pocket sell-side banks, but at privately held firms. They therefore need to keep their positions small and short-lived to keep the capital tied up in margin accounts in check. They trade a lot intradaily and avoid carrying a position overnight. These characterizations suggest that HFTs are best thought of as a new type of intermediary, ..." Menkveld (2013), for instance, studies a large HFT and finds that 4 out of 5 positions are passive. Moreover, the HFT makes money on the spread, but loses money on its positions.

The above observations, combined with the large fixed costs associated with high-frequency trading, suggest that - as a first approximation - our model and analysis may shed some light on electronic limit order markets in which HFTs effectively act as MMs. A proper investigation of information acquisition in such markets is however beyond the scope of our paper. The work of Budish, Cramton and Shim (2015), for example, constitutes a first step in that direction.

\footnotetext{
${ }^{28}$ Even there, traditional designated market makers still play an important role however; see, e.g., ClarkJoseph, Ye and Zi (2017).
} 


\section{Appendix A: Proofs of Sections 3 and 4}

We first introduce some notation. We sometimes write $\bar{\Pi}_{n}(p, q), \Pi_{n}(p, q), \bar{\Pi}_{S}(p, q)$ and $\Pi_{S}(p, q)$ to make explicit that $\bar{\Pi}_{n}, \Pi_{n}, \bar{\Pi}_{S}$ and $\Pi_{S}$ are functions of the (fixed) information acquisition probabilities of the trading game. Furthermore, we let $\bar{\Pi}_{n}(b \mid$ sell $)$ denote $\mathrm{MM} n \mathrm{H}$ 's equilibrium expected profit in the trading game from bidding $b$ conditional on a sell order, ${ }^{29}$ and similarly define $\Pi_{n}(b \mid$ sell $)$ for $\mathrm{MM} n \mathrm{U}$. Finally, let $\gamma:=\mathbb{P}(V=0 \mid$ sell $)$.

Proof of Lemma 1: Follows from Proposition C1, in Online Appendix C.

Proof of Lemma 2: This is part 6 of Lemma C1, in Online Appendix C.

Proof of Theorem 1: Consider $p, q \in(0,1)$ (the other cases are similar). The arguments in the proof of Proposition $\mathrm{C} 1$ establish that a trading equilibrium exists, is unique up to the tie-breaking rule, and satisfies: (i) $\sigma_{1}=\sigma_{2}=\sigma, \underline{\sigma}_{1}=\underline{\sigma}_{2}=\underline{\sigma}$ and $\bar{\sigma}_{1}=\bar{\sigma}_{2}=\bar{\sigma}$; (ii) $\underline{\sigma}(0)=1$; (iii) $\sigma$ and $\bar{\sigma}$ are atomless, with $\operatorname{supp}(\sigma)=[0, l]$ and $\operatorname{supp}(\bar{\sigma})=[l, u]$; (iv) the speculator sells (resp. buys) with probability 1 when she is informed and $V=0$ (resp. $V=1$ ) and abstains when she is uninformed; (v) MMnU's equiprofit condition is

$$
-\gamma[p+(1-p) \sigma(b)] b+(1-\gamma)(1-p) \sigma(b)(1-b)=0, \quad \forall b \in[0, l]
$$

and MM $n$ U's equilibrium expected profit from bidding $l$ conditional on a sell order is

$$
\Pi_{n}(l \mid \text { sell })=-\gamma(p+(1-p) \sigma(l)) l+(1-\gamma) \bar{\Pi}_{n}(l \mid \text { sell }) .
$$

The remarks above imply

$$
\begin{gathered}
\gamma=\frac{\frac{\pi q}{2}+\frac{1-\pi}{4}}{\frac{\pi q}{2}+\frac{1-\pi}{2}}, \\
l=\frac{(1-\gamma)(1-p)}{\gamma+(1-\gamma)(1-p)},
\end{gathered}
$$

\footnotetext{
${ }^{29}$ Notice that by the symmetry of the equilibrium, MM $n \mathrm{H}$ 's equilibrium expected trading profit equals MM $n$ L's equilibrium expected trading profit, and both equal $\bar{\Pi}_{n}$.
} 
and

$$
\bar{\Pi}_{n}(l \mid \text { sell })=\frac{\gamma l}{1-\gamma}
$$

Combining (A4), (A5) and the symmetry between the bid and ask sides of the market then yields ${ }^{30}$

$$
\bar{\Pi}_{n}=\left(\frac{1-\pi}{2}\right)\left(\frac{\gamma(1-p)}{\gamma+(1-\gamma)(1-p)}\right) .
$$

By (A3), $\gamma$ increases with $q$. So, by (A6), $\bar{\Pi}_{n}$ is increasing in $q$ and decreasing in $p$. Since $\Pi_{n}=0$, these observations establish part 2 of the theorem.

We next prove part 1 of the theorem. Since the speculator abstains when she is uninformed, $\Pi_{S}=0$. So our goal is to show that $\bar{\Pi}_{S}$ is decreasing in $p$ and $q$. Observe that, by symmetry of the bid and ask sides of the market,

$$
\bar{\Pi}_{S}=\int b d F(b)
$$

where $F(b):=\mathbb{P}(\hat{b} \leq b \mid V=0)$. As $\underline{\sigma}(0)=1$, we can write

$$
F(b)=(1-p)^{2} \sigma^{2}(b)+2 p(1-p) \sigma(b)+p^{2} .
$$

We proceed to show that an increase in either $p$ or $q$ induces an inverse first-order stochastic dominance shift of $F$. Pick an arbitrary $b \in(0, l)$. First, rearranging (A1) yields

$$
\sigma(b)=\frac{\gamma p b}{(1-p)[(1-\gamma)(1-b)-\gamma b]} .
$$

So $\sigma(b)$ is increasing in $p$. Moreover, as $\sigma(b)$ is increasing in $\gamma$ which itself is increasing in $q$, we conclude that $\sigma(b)$ is also increasing in $q$. Now, differentiating (A8) gives

$$
\begin{aligned}
& \frac{d F(b)}{d p}=2(1-p)^{2} \sigma(b) \frac{d \sigma(b)}{d p}+2 p(1-p) \frac{d \sigma(b)}{d p}+2\left[(1-\sigma(b)) p+(1-p)\left(\sigma(b)-\sigma(b)^{2}\right)\right] \\
& \frac{d F(b)}{d q}=2(1-p)^{2} \sigma(b) \frac{d \sigma(b)}{d q}+2 p(1-p) \frac{d \sigma(b)}{d q}
\end{aligned}
$$

So $\frac{d \sigma(b)}{d p}>0$ implies $\frac{d F(b)}{d p}>0$, while $\frac{d \sigma(b)}{d q}>0$ implies $\frac{d F(b)}{d q}>0$. An increase in either $p$

\footnotetext{
${ }^{30}$ Since $l$ is in the support of $\bar{\sigma}, \mathrm{MM} n \mathrm{H}$ makes expected profit given by (A5) whenever the trader is hit by a liquidity shock and sells the asset. By symmetry, MM $n$ L makes expected profit given by (A5) whenever the trader is hit by a liquidity shock and buys the asset.
} 
or $q$ therefore induces an inverse first-order stochastic dominance shift of $F$. Equation (A7) finishes to show that $\bar{\Pi}_{S}$ is decreasing in $p$ and $q$.

\section{Proof of Theorem 2:}

Step 1: the equilibrium expected profit functions of the trading game (that is, $\bar{\Pi}_{n}, \bar{\Pi}_{S}, \Pi_{n}, \Pi_{S}$ ) are all continuous in $p$ and $q$.

We have $\Pi_{n}=\Pi_{S}=0, \bar{\Pi}_{n}$ given by (A6), and $\bar{\Pi}_{S}$ given by (A7), with (A8) giving $F$ and (A9) giving $\sigma$. Step 1 ensues.

Step 2: there exists an equilibrium.

Define for $i=n, S$ the set-valued functions

$$
\psi_{i}(p, q):=\left\{\begin{array}{lll}
\{0\} & \text { if } & \bar{\Pi}_{i}(p, q)-c<\Pi_{i}(p, q) \\
{[0,1]} & \text { if } & \bar{\Pi}_{i}(p, q)-c=\Pi_{i}(p, q) \\
\{1\} & \text { if } & \bar{\Pi}_{i}(p, q)-c>\Pi_{i}(p, q)
\end{array}\right.
$$

For all $(p, q) \in[0,1] \times[0,1], \psi_{i}(p, q)$ is convex. Next, consider sequences $\left\{p_{k}\right\}_{k \in \mathbb{N}}$ and $\left\{q_{k}\right\}_{k \in \mathbb{N}}$ converging, respectively, to $p^{\dagger}$ and $q^{\dagger}$. Suppose the sequence $\left\{u_{k}\right\}_{k \in \mathbb{N}}$ converges to $u$ and satisfies $u_{k} \in \psi_{i}\left(p_{k}, q_{k}\right)$ for all $k \in \mathbb{N}$. If $\psi_{i}\left(p^{\dagger}, q^{\dagger}\right)=[0,1]$ then $u \in \psi_{i}\left(p^{\dagger}, q^{\dagger}\right)$ is immediate. By Step $1, \Pi_{i}$ and $\bar{\Pi}_{i}$ are both continuous in $p$ and $q$. Therefore $\psi_{i}\left(p^{\dagger}, q^{\dagger}\right)=\{0\}$ implies $\psi_{i}\left(p_{k}, q_{k}\right)=\{0\}$ for all sufficiently large $k$, and $\psi_{i}\left(p^{\dagger}, q^{\dagger}\right)=\{1\}$ implies $\psi_{i}\left(p_{k}, q_{k}\right)=\{1\}$ for all sufficiently large $k$. This shows that $\psi_{i}$ has closed graph. We may therefore apply Kakutani's fixed point theorem to the correspondence $\psi_{n} \times \psi_{S}$. By construction, if $(p, q) \in$ $\left(\psi_{n}(p, q), \psi_{S}(p, q)\right)$, an equilibrium exists in which MMs acquire information with probability $p$ while the speculator acquires information with probability $q$.

Step 3: any equilibrium is a WELM equilibrium.

It follows from Lemma 2 and the remarks in the main text after the lemma that any equilibrium has to be such that both MMs acquire information with the same probability. The other properties are immediate from the arguments in the proof of Proposition $\mathrm{C} 1$, in Online Appendix C. 
Step 4: the equilibrium information acquisition probabilities $p$ and $q$ are uniquely determined. Assume that an equilibrium exists in which MMs acquire information with probability $p \in$ $(0,1)$ while the speculator acquires information with probability $q \in(0,1)$ (the other cases are similar). In the rest of the paragraph, we make repeated use of Theorem 1. Suppose by way of contradiction that an equilibrium exists with information acquisition probabilities $p^{\prime}$ and $q^{\prime}$, where either $p^{\prime} \neq p$ or $q^{\prime} \neq q$. We proceed by cases.

Case 1: $p^{\prime}=p$. If $p^{\prime}=p$ then either $q^{\prime}>q$ or $q^{\prime}<q$. If $q^{\prime}>q$ then $\bar{\Pi}_{S}\left(p^{\prime}, q^{\prime}\right)-\Pi_{S}\left(p^{\prime}, q^{\prime}\right)<$ $\bar{\Pi}_{S}(p, q)-\Pi_{S}(p, q)=c$, contradicting $q^{\prime}>0$. If $q^{\prime}<q$, then $\bar{\Pi}_{S}\left(p^{\prime}, q^{\prime}\right)-\Pi_{S}\left(p^{\prime}, q^{\prime}\right)>\bar{\Pi}_{S}(p, q)-$ $\Pi_{S}(p, q)=c$, contradicting $q^{\prime}<1$. This rules out $p^{\prime}=p$.

Case 2: $p^{\prime}>p$. Then, $\bar{\Pi}_{n}\left(p^{\prime}, q\right)-\Pi_{n}\left(p^{\prime}, q\right)<\bar{\Pi}_{n}(p, q)-\Pi_{n}(p, q) \leq c$. As $p^{\prime}>0$, we also have $\bar{\Pi}_{n}\left(p^{\prime}, q^{\prime}\right)-\Pi_{n}\left(p^{\prime}, q^{\prime}\right) \geq c$. Therefore, $q^{\prime}>q$, which again implies $q<1$. But then, $\bar{\Pi}_{S}\left(p^{\prime}, q^{\prime}\right)-\Pi_{S}\left(p^{\prime}, q^{\prime}\right)<\bar{\Pi}_{S}(p, q)-\Pi_{S}(p, q) \leq c$, contradicting $q^{\prime}>0$. This rules out $p^{\prime}>p$.

Case 3: $p^{\prime}<p$. Then, $\bar{\Pi}_{n}\left(p^{\prime}, q\right)-\Pi_{n}\left(p^{\prime}, q\right)>\bar{\Pi}_{n}(p, q)-\Pi_{n}(p, q) \geq c$. As $p^{\prime}<1$, we also have $\bar{\Pi}_{n}\left(p^{\prime}, q^{\prime}\right)-\Pi_{n}\left(p^{\prime}, q^{\prime}\right) \leq c$. Therefore, $q^{\prime}<q$, which again implies $q>0$. But then, $\bar{\Pi}_{S}\left(p^{\prime}, q^{\prime}\right)-\Pi_{S}\left(p^{\prime}, q^{\prime}\right)>\bar{\Pi}_{S}(p, q)-\Pi_{S}(p, q) \geq c$, contradicting $q^{\prime}<1$. This rules out $p^{\prime}<p$.

The cases above rule out the existence of an equilibrium with information acquisition probabilities $p^{\prime}$ and $q^{\prime}$ different from $p$ and $q$.

Step 5: in equilibrium, $p$ is non-increasing in $c$.

Consider $c_{a}<c_{b}$. Let $p_{a}$ and $q_{a}$ (respectively $p_{b}$ and $q_{b}$ ) denote the equilibrium information acquisition probabilities given $c=c_{a}$ (resp. $c=c_{b}$ ). Suppose by way of contradiction that $p_{b}>p_{a}$. Then

$$
\bar{\Pi}_{n}\left(p_{a}, q_{a}\right)-\Pi_{n}\left(p_{a}, q_{a}\right) \leq c_{a}<c_{b} \leq \bar{\Pi}_{n}\left(p_{b}, q_{b}\right)-\Pi_{n}\left(p_{b}, q_{b}\right) .
$$

Using Theorem 1, this in turn implies $q_{b}>q_{a}$. But then,

$$
\bar{\Pi}_{S}\left(p_{a}, q_{a}\right)-\Pi_{S}\left(p_{a}, q_{a}\right) \geq \bar{\Pi}_{S}\left(p_{b}, q_{b}\right)-\pi_{S}\left(p_{b}, q_{b}\right) \geq c_{b}>c_{a},
$$

contradicting $q_{a}<1$.

Step 6: in equilibrium, $p \rightarrow 1$ as $c \rightarrow 0$.

This step is immediate from the arguments given in the text below the statement of Theorem 2 . 
Step 7: in equilibrium, $q=0$ for all sufficiently small $c$.

Notice first that $p=1$ implies $\bar{\Pi}_{n}=0$. Hence, in equilibrium, $p<1$. This remark combined with Step 6 implies that, in equilibrium, $\bar{\Pi}_{n}-\Pi_{n}=c$ for all sufficiently small $c$ and, as $\Pi_{n}=0, \bar{\Pi}_{n}(p, q)=c$, with $\bar{\Pi}_{n}(p, q)$ given by (A6). Now, by part 2 of Theorem $1, \bar{\Pi}_{n}(p, q)$ is decreasing in $p$ but increasing in $q$. So, for all sufficiently small $c$, in equilibrium: $p \geq p^{*}(c)$, where $p^{*}(c)$ is defined implicitly by $\bar{\Pi}_{n}\left(p^{*}(c), 0\right)=c$. Using (A6) yields

$$
p^{*}(c)=\frac{1-\pi-4 c}{1-\pi-2 c} .
$$

Next, the remark that $p \geq p^{*}(c)$ at sufficiently small $c$ combined with part 1 of Theorem 1 shows that, in equilibrium, for all sufficiently small $c$ :

$$
\bar{\Pi}_{S}(p, q)-\Pi_{S}(p, q) \leq \bar{\Pi}_{S}\left(p^{*}(c), 0\right)-\Pi_{S}\left(p^{*}(c), 0\right)=\bar{\Pi}_{S}\left(p^{*}(c), 0\right) .
$$

Let $l(p, q)$ be given by (A4) and (A3). The informed speculator who observed $v=0$ will find an uninformed MM to sell the asset to with probability $2 p(1-p)+(1-p)^{2}$ and will at most obtain the price $l(p, q)$. Since $p \geq p^{*}(c)$ and given that $l(p, q)$ is decreasing in $p$ and $q$, we can place an upper bound on the speculator's gain from being informed:

$$
\begin{aligned}
\bar{\Pi}_{S}(p, q)-\Pi_{S}(p, q) & \leq\left[2 p^{*}(c)\left(1-p^{*}(c)\right)+\left(1-p^{*}(c)\right)^{2}\right] l\left(p^{*}(c), 0\right) \\
& =\left(1-p^{*}(c)\right)\left(p^{*}(c)+1\right) l\left(p^{*}(c), 0\right) \\
& \leq 2\left(1-p^{*}(c)\right) l\left(p^{*}(c), 0\right)
\end{aligned}
$$

Substituting (A10) into (A4) yields

$$
l\left(p^{*}(c), 0\right)=\frac{2 c}{1-\pi} .
$$

Combining (A10), (A11) and (A12) shows that for all sufficiently small $c$, in equilibrium:

$$
\bar{\Pi}_{S}(p, q)-\Pi_{S}(p, q) \leq \frac{8 c^{2}}{(1-\pi)(1-\pi-2 c)} .
$$

Hence, in equilibrium, $\bar{\Pi}_{S}(p, q)-\Pi_{S}(p, q)<c$ for all $c$ sufficiently small, concluding the proof of Step 7. 
Step 8: there exist $0<\underline{c}<\bar{c}<\frac{1}{2}$ such that, in equilibrium, $q=0$ if and only if $c \leq \underline{c}$ and $p=0$ if and only if $c \geq \bar{c}$.

Follows from Lemmata A1 and A2 below.

Lemma A1. Let $c \in\left(0, \frac{1}{2}\right)$. Then, in equilibrium, the following are equivalent:

(i) $p=0$;

(ii) $p=0<q$;

(iii) $c \geq \bar{c}(\pi)=\frac{1-\pi^{2}}{4}$.

Proof: Fix $p=0$ and $q=1$, and consider the resulting trading game. With both MMs uninformed, Bertrand competition yields $\hat{b}=\mathbb{E}[V \mid$ sell $]=\frac{1-\pi}{2}=1-\hat{a}$. Hence,

$$
\bar{\Pi}_{S}(0,1)-\Pi_{S}(0,1)=\frac{1}{2} \hat{b}+\frac{1}{2}(1-\hat{a})=\frac{1-\pi}{2} .
$$

On the other hand,

$$
\bar{\Pi}_{n}(0,1)-\Pi_{n}(0,1)=(1-\pi)\left(\frac{1}{4}(1-\hat{b})+\frac{1}{4} \hat{a}\right)=\frac{1-\pi^{2}}{4} .
$$

Therefore, for $c \in\left[\frac{1-\pi^{2}}{4}, \frac{1-\pi}{2}\right]$, the equilibrium information acquisition probabilities are $p=0$ and $q=1$. A similar argument establishes that for $c \in\left(\frac{1-\pi}{2}, \frac{1}{2}\right)$ the equilibrium information acquisition probabilities are $p=0$ and $q \in(0,1)$.

We next show that in equilibrium $p>0$ for all $c<\frac{1-\pi^{2}}{4}$. Suppose $c<\frac{1-\pi^{2}}{4}$. First, notice that part 1 of Theorem 1 combined with the derivation of the previous paragraph yields $\bar{\Pi}_{S}(0, q)-\Pi_{S}(0, q) \geq \frac{1-\pi}{2}$ for all $q \in[0,1]$. So if in equilibrium $p=0$ then $q=1$. Yet we saw above that $\bar{\Pi}_{n}(0,1)-\Pi_{n}(0,1)=\frac{1-\pi^{2}}{4}$. The latter observation rules out the possibility of $p=0$ in equilibrium.

Lemma A2. Let $c \in\left(0, \frac{1}{2}\right)$. Then there exists a monotone decreasing function $\underline{\pi}(\cdot)$ such that in equilibrium $q=0$ if and only if $\pi \leq \underline{\pi}(c)$.

Proof: Throughout the proof we restrict attention to $c<\frac{1}{2}$. 
Step 1: if in equilibrium $q=0$ for a given value of $\pi$ then in equilibrium $q=0$ as well for all smaller values of $\pi$.

Consider $\pi_{b}<\pi_{a}$. Let $p_{a}$ and $q_{a}$ (respectively $p_{b}$ and $q_{b}$ ) denote the equilibrium information acquisition probabilities given $\pi=\pi_{a}$ (resp. $\pi=\pi_{b}$ ). Suppose that $q_{a}=0$. We will show that $q_{b}=0$ as well. First, (A6) yields

$$
\bar{\Pi}_{n}(p, 0 ; \pi)-\Pi_{n}(p, 0 ; \pi)=\left(\frac{1-\pi}{2}\right)\left(\frac{1-p}{2-p}\right) .
$$

By Lemma A1, $p_{a}>0$. Since in equilibrium MMs always acquire information with probability less than $1, p_{a} \in(0,1)$. Therefore, using $\bar{\Pi}_{n}\left(p_{a}, 0 ; \pi_{a}\right)-\Pi_{n}\left(p_{a}, 0 ; \pi_{a}\right)=c$ and solving for $p_{a}$ gives

$$
p_{a}=\frac{1-\pi_{a}-4 c}{1-\pi_{a}-2 c} .
$$

Now define

$$
p_{b}^{\prime}:=\frac{1-\pi_{b}-4 c}{1-\pi_{b}-2 c} .
$$

Note that $p_{b}^{\prime}>p_{a}$, since $\pi_{b}<\pi_{a}$. Moreover,

$$
\left(\frac{1-\pi_{b}}{2}\right)\left(\frac{1-p_{b}^{\prime}}{2-p_{b}^{\prime}}\right)=c
$$

Thus, by (A13),

$$
\bar{\Pi}_{n}\left(p_{b}^{\prime}, 0 ; \pi_{b}\right)-\Pi_{n}\left(p_{b}^{\prime}, 0 ; \pi_{b}\right)=c .
$$

On the other hand, observe that fixing $q=0$, the speculator's expected profit functions $\bar{\Pi}_{S}$ and $\Pi_{S}$ in the trading game are independent of $\pi$, since for $q=0$ none of the price distributions depend on $\pi$. This remark, combined with part 1 of Theorem 1, yields

$$
\bar{\Pi}_{S}\left(p_{b}^{\prime}, 0 ; \pi_{b}\right)-\Pi_{S}\left(p_{b}^{\prime}, 0 ; \pi_{b}\right)<\bar{\Pi}_{S}\left(p_{a}, 0 ; \pi_{a}\right)-\Pi_{S}\left(p_{a}, 0 ; \pi_{a}\right) \leq c .
$$

It now follows from (A14) and (A15) that $p_{b}^{\prime}$ and 0 are the equilibrium information acquisition probabilities given $\pi=\pi_{b}$. This concludes the proof of Step 1 .

In what follows, let

$$
p^{*}(c, \pi):=\frac{1-\pi-4 c}{1-\pi-2 c} .
$$


We also define

$$
\underline{\pi}(c):=\max \{0, \sup \{\pi: q=0 \text { in equilibrium }\}\} .
$$

Step 2: $\bar{\Pi}_{n}\left(p^{*}(c, \pi), 0\right)=c$.

Immediate from $(\mathrm{A} 6)$.

Step 3: $\underline{\pi}(c)>0$ implies $\bar{\Pi}_{S}\left(p^{*}(c, \underline{\pi}(c)), 0\right)=c$.

Suppose $\underline{\pi}(c)>0$. Then we can find a sequence $\left\{\pi_{k}\right\}_{k \in \mathbb{N}}$, with limit $\underline{\pi}(c)$, such that in equilibrium: $\pi=\pi_{k}$ implies $q=q_{k}=0$. Hence, by Lemma A1, for all $k$ sufficiently large, in equilibrium: $\pi=\pi_{k}$ implies $p=p_{k}>0$. Therefore, $\bar{\Pi}_{n}\left(p_{k}, 0\right)=\bar{\Pi}_{n}\left(p_{k}, 0\right)-\Pi_{n}\left(p_{k}, 0\right)=c$ for all $k$ sufficiently large. We conclude, using Step 2 and the monotonicity of $\bar{\Pi}_{n}$ with respect to $p$, that $p_{k}=p^{*}\left(c, \pi_{k}\right)$ for all $k$ sufficiently large. As $\bar{\Pi}_{S}\left(p_{k}, 0\right) \leq c$ irrespective of $k$, we find that $\bar{\Pi}_{S}\left(p^{*}\left(c, \pi_{k}\right), 0\right) \leq c$ for all $k$ sufficiently large. Now, we saw in Step 1 of the proof of Theorem 2 that $\bar{\Pi}_{S}$ is continuous in $p$. Moreover, (A16) shows that $p^{*}$ is continuous in $\pi$. Hence, $\bar{\Pi}_{S}\left(p^{*}(c, \underline{\pi}(c)), 0\right) \leq c$. A similar argument rules out $\bar{\Pi}_{S}\left(p^{*}(c, \underline{\pi}(c)), 0\right)<c$.

Step 4: $\underline{\pi}(c)>0$ implies $\underline{\pi}(c)<1-4 c$.

Suppose $\underline{\pi}(c)>0$. Reasoning as in Step 3 establishes that $p^{*}(c, \underline{\pi}(c)) \geq 0$. Hence, by (A16), $\underline{\pi}(c) \leq 1-4 c$. Next, suppose by way of contradiction that $\underline{\pi}(c)=1-4 c$. Then, $p^{*}(c, \underline{\pi}(c))=0$. Hence, by Step 3: $\bar{\Pi}_{S}(0,0)=c$. But that is impossible, since $\bar{\Pi}_{S}(0,0)=\frac{1}{2}$ whereas $c<\frac{1}{2}$.

Step 5: $\underline{\pi}($.$) is continuous.$

Step 5 follows from Step 3 and the remarks that $\bar{\Pi}_{S}$ is continuous in $p$ while $p^{*}$ is continuous in both of its arguments.

Step 6: $\underline{\pi}\left(c_{a}\right)>0$ implies $\underline{\pi}\left(c_{b}\right)<\underline{\pi}\left(c_{a}\right)$ for all $c_{b} \in\left(c_{a}, \frac{1}{2}\right)$.

Suppose by way of contradiction that there exist $0<c_{a}<c_{b}<\frac{1}{2}$ with $0<\underline{\pi}\left(c_{a}\right) \leq \underline{\pi}\left(c_{b}\right)$. Then, combining Steps 4 and 5 implies the existence of $0<c_{a}<c_{b}^{\prime}<\frac{1}{2}$ with $0<\underline{\pi}\left(c_{a}\right)=\underline{\pi}\left(c_{b}^{\prime}\right)$. By Steps 4 and 5, the function

$$
H(c):=\bar{\Pi}_{S}\left(p^{*}\left(c, \underline{\pi}\left(c_{a}\right)\right), 0\right)-c
$$


thus crosses the horizontal axis at least twice within the open interval $\left(0, \frac{1-\underline{\pi}\left(c_{a}\right)}{4}\right)$, once at $c=c_{a}$ and once at $c=c_{b}^{\prime}$, contradicting Lemma C2 in Online Appendix C.

Proof of Proposition 1: Combining Lemmata A1 and A2 establishes the main part of the proposition. We prove the remaining parts below.

Claim: the equilibrium $q$ tends to 0 as $\pi$ tends to 1 .

Suppose by way of contradiction that the equilibrium probability $q$ with which the speculator acquires information does not tend to 0 as $\pi$ tends to 1 . Then we can find $\varepsilon>0$ and a sequence $\left\{\pi_{k}\right\}_{k \in \mathbb{N}}$ with limit 1 such that each element in the equilibrium sequence $q_{k}$ is greater than $\varepsilon$. Therefore, by (A3), $\gamma_{k}$ converges to 1 and, by (A4), $l_{k}$ converges to 0 . Yet, by symmetry of the bid and ask sides of the market:

$$
\bar{\Pi}_{S}\left(p_{k}, q_{k}\right)-\Pi_{S}\left(p_{k}, q_{k}\right)=\bar{\Pi}_{S}\left(p_{k}, q_{k}\right) \leq l_{k}
$$

Therefore, $\bar{\Pi}_{S}\left(p_{k}, q_{k}\right)-\Pi_{S}\left(p_{k}, q_{k}\right)<c$ for all sufficiently large $k$, contradicting $q_{k}>\varepsilon$.

Claim: the equilibrium probability $p$ with which a MM acquires information is non-increasing in $\pi$.

First, using (A6) gives us

$$
\frac{\partial^{2} \bar{\Pi}_{n}(p, q)}{\partial \pi^{2}}=-\frac{4(1-p)^{2} q^{2}}{[(2-p)(1-\pi)+2 \pi q]^{3}} \leq 0
$$

On the other hand,

$$
\left.\frac{\partial \bar{\Pi}_{n}(p, q)}{\partial \pi}\right|_{\pi=0}=-\frac{(1-p)[2-2 q+p(2 q-1)]}{2(2-p)^{2}} \leq 0 .
$$

We therefore obtain

$$
\frac{\partial \bar{\Pi}_{n}(p, q)}{\partial \pi} \leq 0
$$

Next, by (A9), $\frac{\partial \sigma(b)}{\partial \gamma} \geq 0$. As $\frac{\partial \gamma}{\partial \pi} \geq 0$, we obtain $\frac{\partial \sigma(b)}{\partial \pi} \geq 0$. This implies, by (A7) and (A8), that

$$
\frac{\partial \bar{\Pi}_{S}(p, q)}{\partial \pi} \leq 0
$$


Now consider $\pi_{b}>\pi_{a}$. Let $p_{a}$ and $q_{a}$ (resp. $p_{b}$ and $q_{b}$ ) denote the equilibrium information acquisition probabilities given $\pi=\pi_{a}$ (resp. $\pi=\pi_{b}$ ). Suppose by way of contradiction that $p_{b}>p_{a}$. Then

$$
\bar{\Pi}_{n}\left(p_{b}, q_{b} ; \pi_{b}\right) \geq c \geq \bar{\Pi}_{n}\left(p_{a}, q_{a} ; \pi_{a}\right) .
$$

As $\pi_{b}>\pi_{a}$ and $p_{b}>p_{a}$, we conclude by (A17) and part 2 of Theorem 1 that $q_{b}>q_{a}$. But then (A18) and part 1 of Theorem 1 give

$$
\bar{\Pi}_{S}\left(p_{b}, q_{b} ; \pi_{b}\right)<\bar{\Pi}_{S}\left(p_{a}, q_{a} ; \pi_{a}\right),
$$

contradicting $q_{b}>q_{a}$. 


\section{Appendix B: Proofs of Section 6}

We first introduce some notation. We sometimes write $\bar{\Pi}_{n}(p, q ; z), \Pi_{n}(p, q ; z), \bar{\Pi}_{S}(p, q ; z)$ and $\Pi_{S}(p, q ; z)$ to make explicit that $\bar{\Pi}_{n}, \Pi_{n}, \bar{\Pi}_{S}$ and $\Pi_{S}$ are functions of the (fixed) information acquisition probabilities of the trading game, and of the probability that the quotes are observed.

Proof of Proposition 2: By Proposition D1, in any WELM trading equilibrium:

$$
\bar{\Pi}_{n}(p, q ; z)=\left(\frac{(1-\pi)(1-p)}{2}\right) \frac{1-\pi(1-2 q)+2 \pi p(1-q) z}{2-p-2 \pi(1-q)+\pi p(1+2(1-q) z)},
$$

and

$$
\sigma(b)=\frac{(1+\pi(2 z-1)+2 \pi q(1-z)) p b}{(1-p)(1-\pi-2 b(1-\pi(1-q)))}, \quad \forall b \in[0, l] .
$$

Differentiating (B1) with respect to $z$ gives

$$
\frac{\partial \bar{\Pi}_{n}(p, q ; z)}{\partial z}=\frac{(1-p)^{2} p(1-\pi)^{2} \pi(1-q)}{(2-p-2 \pi(1-q)+\pi p(1+2(1-q) z))^{2}}>0 .
$$

As $\Pi_{n}(p, q ; z)=0$ in a WELM trading equilibrium (see Online Appendix D), MMn's gain from becoming informed therefore increases in $z$.

Next, in any WELM trading equilibrium:

$$
\bar{\Pi}_{S}(p, q ; z)=2 p(1-p) \int_{0}^{l} b d \sigma(b)+(1-p)^{2} \int_{0}^{l} b d \sigma^{2}(b) .
$$

If both MMs are informed then the informed speculator makes zero profit. With probability $2(1-p) p$ one MM is informed and the other is uninformed. In this case, by symmetry of the bid and ask sides of the market, the informed speculator's expected profit equals the expected bid of the uninformed MM. With probability $(1-p)^{2}$ both MMs are uninformed. In this case, by symmetry of the bid and ask sides of the market, the informed speculator's expected profit equals the expected maximum bid of the uninformed MMs. On the other hand,

$$
\Pi_{S}(p, q ; z)=2 p(1-p) z \int_{0}^{l} b d \sigma(b)
$$

If the speculator does not acquire information, $\mathrm{MM} n$ is informed, quotes are observable and 
$\mathrm{MM} m$ is uninformed then the speculator learns $v$ from MMn's quotes and makes profit from trading with MMm. Thus, the speculator's gain from acquiring information is

$$
\bar{\Pi}_{S}(p, q ; z)-\Pi_{S}(p, q ; z)=2(1-z) p(1-p) \int_{0}^{l} b d \sigma(b)+(1-p)^{2} \int_{0}^{l} b d \sigma^{2}(b) .
$$

Now, by $(\mathrm{B} 2), \sigma(b)$ is increasing in $z$. So the right-hand side of (B3) decreases in $z$.

Proposition B1. Let $z=1$. There exists $\hat{p}<1$, independent of $q$, such that, for all $p>\hat{p}$, $\bar{\Pi}_{n}-\Pi_{n}$ is decreasing in $p$. There exists $\hat{q}<1$ such that, for all $q>\hat{q}, \bar{\Pi}_{n}-\Pi_{n}$ is decreasing in $p$. If $\pi>\frac{1}{3}$ then, for $p$ and $q$ sufficiently small, $\bar{\Pi}_{n}-\Pi_{n}$ is increasing in $p$.

Proof: Taking $z=1$ and differentiating (B1) with respect to $p$ gives

$$
\frac{\partial \bar{\Pi}_{n}(p, q ; 1)}{\partial p}=\frac{(1-\pi) A(p, q)}{2(2 \pi(p-1) q-3 \pi p+p+2 \pi-2)^{2}}
$$

where $A(p, q)$ is a continuous function of $p$ and $q$ that takes the values $A(0,0)=(1-\pi)(3 \pi-$ $1)$, and $A(1, q)=A(p, 1)=-(1+\pi)^{2}$. Continuity of the partial derivative together with $\Pi_{n}(p, q ; z)=0$ then yields the desired result.

Proof of Theorem 3: In any WELM trading equilibrium, $\bar{\Pi}_{n}$ is given by (B1). Taking the derivative with respect to $q$ :

$$
\frac{\partial \bar{\Pi}_{n}(p, q ; z)}{\partial q}=\frac{(1-p)^{2}(1-\pi)^{2} \pi(1-z p)}{(2(1-\pi(1-q))+2 \pi p z(1-q)-p(1-\pi))^{2}}>0 .
$$

As $\Pi_{n}(p, q ; z)=0, \mathrm{MM} n$ 's gain from becoming informed therefore increases in $q$. The comparative statics result relative to $p$ for $\mathrm{MM} n$ follows from Proposition B1. For the comparative statics results pertaining to the speculator, notice that by (B2), $\sigma(b)$ is increasing in $p$ and $q$. So the right-hand side of (B3) decreases in $p$ and $q$.

Proof of Theorem 4: Suppose $z=1$ (the proof for the case $z \in(0,1)$ is almost identical). In the rest of the proof, let $p_{0}$ (resp. $q_{0}$ ) denote MMs' (resp. the speculator's) unique equilibrium information acquisition probability in any equilibrium with $z=0$. Let $\underline{c}$ and $\bar{c}$ denote the cutoffs from Theorem 2. 
Step 1: there exists $c^{\dagger}$ such that, for all $c<c^{\dagger}$, a WELM equilibrium exists and satisfies $p \geq p_{0}$ and $q=0$.

Recall: (a) $p_{0}$ tends to 1 as $c$ tends to 0 , and (b) by Proposition D3, a WELM trading equilibrium exists for all $p \geq \frac{\sqrt{2 \pi}}{\sqrt{2 \pi}+\sqrt{1-\pi}}$. Pick $\tilde{c}>0$ such that $p_{0}>\frac{\sqrt{2 \pi}}{\sqrt{2 \pi}+\sqrt{1-\pi}}$ for all $c<\tilde{c}$. Consider $c<\min \{\tilde{c}, \underline{c}\}$. Then, using Proposition 2,

$$
\bar{\Pi}_{n}\left(p_{0}, 0 ; 1\right)-\Pi_{n}\left(p_{0}, 0 ; 1\right)>\bar{\Pi}_{n}\left(p_{0}, 0 ; 0\right)-\Pi_{n}\left(p_{0}, 0 ; 0\right) \geq c .
$$

Next, as $\bar{\Pi}_{n}(1,0 ; 1)-\Pi_{n}(1,0 ; 1)=0$, the intermediate value theorem gives $p^{*}>p_{0}$ solving

$$
\bar{\Pi}_{n}\left(p^{*}, 0 ; 1\right)-\Pi_{n}\left(p^{*}, 0 ; 1\right)=c .
$$

As $p^{*}>p_{0}$, Proposition 2 and Theorem 3 give

$$
\bar{\Pi}_{S}\left(p^{*}, 0 ; 1\right)-\Pi_{S}\left(p^{*}, 0 ; 1\right)<\bar{\Pi}_{S}\left(p_{0}, 0 ; 0\right)-\Pi_{S}\left(p_{0}, 0 ; 0\right) \leq c .
$$

A WELM equilibrium therefore exists, with $p=p^{*}$ and $q=0$.

Step 2: for all $\hat{p}<1$, there exists $\hat{c}>0$ such that, in any equilibrium, $p>\hat{p}$ whenever $c<\hat{c}$.

As uninformed MMs never set bid prices above $\frac{1}{2}$ nor set ask prices below $\frac{1}{2}$, MMn's gain from being informed in the trading game is at least as large as $\frac{(1-p)(1-\pi)}{4}$. Thus, in equilibrium, $\frac{(1-p)(1-\pi)}{4} \leq c$.

Step 3: there exists $\hat{c}>0$ such that, in any WELM equilibrium, $p \geq p_{0}$ for all $c<\hat{c}$.

Choose $\hat{p}<1$ such that $\bar{\Pi}_{n}-\Pi_{n}$ is decreasing in $p$ for all $p>\hat{p}$ (such a $\hat{p}$ exists, by Proposition B1). Now pick $\hat{c}$ such that, for all $c<\hat{c}$ : (a) $q_{0}=0$ and (b) $p>\hat{p}$ in any WELM equilibrium (such a $\hat{c}$ exists, by virtue of Theorem 2 combined with Step 2 of the proof). Let $c<\hat{c}$ and suppose by way of contradiction that a WELM equilibrium exists satisfying $\hat{p}<p<p_{0}$. Then $\bar{\Pi}_{n}(p, q ; 1)-\Pi_{n}(p, q ; 1)=c$. We have on the other hand, $\bar{\Pi}_{n}\left(p_{0}, 0 ; 0\right)-\Pi_{n}\left(p_{0}, 0 ; 0\right) \geq c$. Yet, our choice of $\hat{p}$ combined with Proposition 2 and Theorem 3 implies

$$
\bar{\Pi}_{n}(p, q ; 1)-\Pi_{n}(p, q ; 1)>\bar{\Pi}_{n}\left(p_{0}, 0 ; 0\right)-\Pi_{n}\left(p_{0}, 0 ; 0\right),
$$

which clearly cannot be. 
Step 4: in any WELM equilibrium, $q=0$ for all $c<\hat{c}$.

Recall, we chose $\hat{c}$ such that $q_{0}=0$ for all $c<\hat{c}$. So Step 4 is immediate from Step 3 combined with the fact that, by Proposition 2 and Theorem $3, \bar{\Pi}_{S}-\Pi_{S}$ is decreasing in $p, q$ and $z$.

Step 5: for $c \in\left(\frac{(1-\pi)(1+\pi)}{4}, \frac{1}{2}\right)$, a WELM equilibrium exists and satisfies $p=0<q$.

Theorem 2 and the observation made in the text that $\bar{c}=\frac{(1-\pi)(1+\pi)}{4}$ give $p_{0}=0<$ $q_{0}$ for all $c \in\left(\frac{(1-\pi)(1+\pi)}{4}, \frac{1}{2}\right)$. Proposition D3 assures the existence of a WELM trading equilibrium whenever $p=0$. The existence of a WELM equilibrium satisfying $p=p_{0}, q=q_{0}$, $\bar{\Pi}_{n}\left(p_{0}, q_{0} ; 1\right)=\bar{\Pi}_{n}\left(p_{0}, q_{0} ; 0\right), \bar{\Pi}_{S}\left(p_{0}, q_{0} ; 1\right)=\bar{\Pi}_{S}\left(p_{0}, q_{0} ; 0\right), \Pi_{n}\left(p_{0}, q_{0} ; 1\right)=\Pi_{n}\left(p_{0}, q_{0} ; 0\right)$, and $\Pi_{S}\left(p_{0}, q_{0} ; 1\right)=\Pi_{S}\left(p_{0}, q_{0} ; 0\right)$ is now straightforward to verify.

Step 6: for $c \in\left(\frac{1-\pi}{2}, \frac{1}{2}\right), p=0<q$ in any WELM equilibrium.

Recall, $c>\bar{c}=\frac{(1-\pi)(1+\pi)}{4}$ implies $p_{0}=0<q_{0}$. Let $c \in\left(\frac{1-\pi}{2}, \frac{1}{2}\right)$. In any WELM equilibrium, MMs' gain from acquiring information is bounded above by $\frac{1-\pi}{2}$. So $p=0$ in any WELM equilibrium. Moreover, combining Theorems 2 and 3 implies that, for all $q<q_{0}$ :

$$
\bar{\Pi}_{S}(0, q ; 1)-\Pi_{S}(0, q ; 1)>\bar{\Pi}_{S}\left(0, q_{0} ; 1\right)-\Pi_{S}\left(0, q_{0} ; 1\right)=\bar{\Pi}_{S}\left(0, q_{0} ; 0\right)-\Pi_{S}\left(0, q_{0} ; 0\right)=c .
$$

We conclude that $q \geq q_{0}$ in any WELM equilibrium. 


\section{Appendix C: Trading Game of Baseline Model (for online publication)}

In this appendix we analyze the trading game induced by the baseline model. Specifically, throughout this appendix $p_{1}, p_{2}$ and $q$ play the role of parameters: $\mathrm{MM} n$ is informed with probability $p_{n}$, and the speculator is informed with probability $q$. A strategy of $\mathrm{MM} n$ comprises cumulative distribution functions $\sigma_{n}, \underline{\sigma}_{n}$ and $\bar{\sigma}_{n}$ specifying respectively the distribution of the bid price $b_{n}$ of $\mathrm{MM} n \mathrm{U}, \mathrm{MM} n \mathrm{~L}$ and $\mathrm{MM} n \mathrm{H}$. We assume in line with the baseline model that, conditional on $\mathrm{MM} n \mathrm{U}, 1-a_{n}$ is distributed like $b_{n}$. Similarly, we assume that the law of $1-a_{n}$ conditional on $\mathrm{MM} n \mathrm{~L}$ (resp. $\mathrm{MM} n \mathrm{H}$ ) is the same as the law of $b_{n}$ conditional on $\mathrm{MM} n \mathrm{H}$ (resp. $\mathrm{MM} n \mathrm{~L}$ ). A strategy of the speculator specifies her market order as a function of the information she possesses at that point.

The following notation will be used throughout:

- $\Pi_{n}(b \mid$ sell $)$ (respectively $\bar{\Pi}_{n}\left(b \mid\right.$ sell) and $\underline{\Pi}_{n}(b \mid$ sell $)$ ) for MMnU's (resp. MMnH's and $\mathrm{MM} n \mathrm{~L}$ 's) expected trading profit conditional on a sell order, given $b_{n}=b$;

- $\sigma_{n}(b):=\mathbb{P}\left(b_{n} \leq b \mid \mathrm{MM} n \mathrm{U}\right), \bar{\sigma}_{n}(b):=\mathbb{P}\left(b_{n} \leq b \mid \mathrm{MM} n \mathrm{H}\right)$ and $\underline{\sigma}_{n}(b):=\mathbb{P}\left(b_{n} \leq b \mid \mathrm{MM} n \mathrm{~L}\right)$;

- $\Sigma_{n}:=\operatorname{supp}\left(\sigma_{n}\right)$ and $\bar{\Sigma}_{n}:=\operatorname{supp}\left(\bar{\sigma}_{n}\right)$;

- $A_{n}$ (respectively $\bar{A}_{n}$ ) for the set of atoms in MMnU's (resp. MMnH's) strategy;

- $l_{n}:=\sup \Sigma_{n}$

- $\gamma:=\mathbb{P}(V=0 \mid$ sell $)$.

Lemma C1. If $p_{1}=p_{2}=1$ then any trading equilibrium has $a_{1}=a_{2}=b_{1}=b_{2}=V$. If $p_{1}=p_{2}=0$ then $a_{1}=a_{2}=\frac{1-\pi(1-2 q)}{2-2 \pi(1-q)}$ and $b_{1}=b_{2}=\frac{1-\pi}{2-2 \pi(1-q)}$. Otherwise, any trading equilibrium satisfies the following properties:

1. $\underline{\sigma}_{1}(0)=\underline{\sigma}_{2}(0)=1$;

2. $\Sigma_{1} \cup \bar{\Sigma}_{1}=\Sigma_{2} \cup \bar{\Sigma}_{2}=[0, u]$, where $u \in(0,1)$;

3. $A_{1} \cup \bar{A}_{1} \cup A_{2} \cup \bar{A}_{2} \subseteq\{0\}$;

4. if $p_{m} \in(0,1)$ then $\Sigma_{m} \cap \bar{\Sigma}_{m}=\left\{l_{m}\right\}$ and $l_{m}<u$, with $l_{m}>0$ if and only if $p_{n}<1$; 
5. if $1>p_{n} \geq p_{m}>0$ then $\mathbb{E}[V \mid$ sell $]>l_{m} \geq l_{n}>0$, with $l_{m}>l_{n}$ if $p_{n}>p_{m}$;

6. if $p_{n}>p_{m}$ then $0=\Pi_{m}<\Pi_{n}<\bar{\Pi}_{n}=\bar{\Pi}_{m}$.

Proof: The cases $p_{1}=p_{2}=1$ and $p_{1}=p_{2}=0$ are trivial. We prove below that, in any trading equilibrium, properties 1-6 hold in the case $\min \left\{p_{1}, p_{2}\right\} \in(0,1)$, that is, when both market MMs acquire information with positive probability but neither of them becomes informed with probability 1 ; the proof for the case $p_{m}=0<p_{n}$ is similar.

Step 1: $\underline{\sigma}_{1}(0)=\underline{\sigma}_{2}(0)=1$. Suppose by way of contradiction that $\underline{\sigma}_{1}(0)<1$. Then we can find $b^{\prime}>0$ with $b^{\prime} \in \arg \max _{b} \underline{\Pi}_{1}(b \mid$ sell $)$ and $\mathbb{P}\left(b_{1} \geq b^{\prime} \mid\right.$ MM1L $)>0$. The previous remarks imply $\mathbb{P}\left(b_{1}=b^{\prime}\right.$ wins $\left.\mid V=0\right)=0$, for otherwise $\underline{\Pi}_{1}\left(b^{\prime} \mid V=0\right)=-\mathbb{P}\left(b_{1}=b^{\prime}\right.$ wins $\left.\mid V=0\right) b^{\prime}<$ $0=\underline{\Pi}_{1}(0 \mid V=0)$. Next, $\mathbb{P}\left(b_{1}=b^{\prime}\right.$ wins $\left.\mid V=0\right)=0$ implies the existence of $b^{\prime \prime} \geq b^{\prime}$ with $b^{\prime \prime} \in \arg \max _{b} \underline{\Pi}_{2}(b \mid$ sell $)$ and $\mathbb{P}\left(b_{2}=b^{\prime \prime}\right.$ wins $\left.\mid V=0\right)>0$. We therefore obtain $\underline{\Pi}_{2}\left(b^{\prime \prime} \mid\right.$ sell $)<$ $0=\underline{\Pi}_{2}(0 \mid$ sell $)$, which cannot be.

Step 2: $p_{n} \in(0,1) \Rightarrow l_{n} \leq \inf \bar{\Sigma}_{n}$. Suppose by way of contradiction that $l_{n}>\inf \bar{\Sigma}_{n}$. Then we can find $b^{\prime \prime}>b^{\prime}$ with $b^{\prime \prime} \in \arg \max _{b} \Pi_{n}(b \mid$ sell $)$ and $b^{\prime} \in \arg \max _{b} \bar{\Pi}_{n}(b \mid$ sell $)$. Next,

$$
\begin{aligned}
\Pi_{n}\left(b^{\prime \prime} \mid \text { sell }\right) & =-\gamma \mathbb{P}\left(b_{n}=b^{\prime \prime} \text { wins } \mid V=0\right) b^{\prime \prime}+(1-\gamma) \mathbb{P}\left(b_{n}=b^{\prime \prime} \text { wins } \mid V=1\right)\left(1-b^{\prime \prime}\right) \\
& =-\gamma \mathbb{P}\left(b_{n}=b^{\prime \prime} \text { wins } \mid V=0\right) b^{\prime \prime}+(1-\gamma) \bar{\Pi}_{n}\left(b^{\prime \prime} \mid \text { sell }\right) \\
& <-\gamma \mathbb{P}\left(b_{n}=b^{\prime \prime} \text { wins } \mid V=0\right) b^{\prime}+(1-\gamma) \bar{\Pi}_{n}\left(b^{\prime \prime} \mid \text { sell }\right) \\
& \leq-\gamma \mathbb{P}\left(b_{n}=b^{\prime} \text { wins } \mid V=0\right) b^{\prime}+(1-\gamma) \bar{\Pi}_{n}\left(b^{\prime} \mid \text { sell }\right) \\
& =\Pi_{n}\left(b^{\prime} \mid \text { sell }\right)
\end{aligned}
$$

The last inequality holds since $b^{\prime}<b^{\prime \prime}$ and $\mathbb{P}\left(b_{n}\right.$ wins $\left.\mid V=0\right)$ is non-decreasing in $b_{n}$. Thus $\Pi_{n}\left(b^{\prime} \mid\right.$ sell $)>\Pi_{n}\left(b^{\prime \prime} \mid\right.$ sell $)=\max _{b} \Pi_{n}(b \mid$ sell $)$, which cannot be.

Step 3: $0<\sup \bar{\Sigma}_{1}=\sup \bar{\Sigma}_{2}<1$. We start by showing that $\sup \bar{\Sigma}_{1}=\sup \bar{\Sigma}_{2}$. Suppose by way of contradiction that this is not the case, say $u_{n}>u_{m}$, where $u_{n}=\sup \bar{\Sigma}_{n}$ and $u_{m}=\sup \bar{\Sigma}_{m}$. Then, since increasing the bid beyond $u_{m}+\varepsilon$ does not increase the winning probability for $n$, $\exists \varepsilon>0$ such that

$$
\bar{\Pi}_{n}\left(u_{m}+\epsilon \mid \text { sell }\right)>\bar{\Pi}_{n}\left(u_{n}-x \mid \text { sell }\right), \quad \forall x \in[0, \varepsilon],
$$

contradicting $u_{n} \in \bar{\Sigma}_{n}$. Hence, $u_{n}=u_{m}$. Next, let $u$ denote the common supremum; we claim 
that $u \in(0,1)$. Suppose by way of contradiction that $u=0$. One of the two MMs does not win with probability 1 conditional on a tie at 0 , say $\mathbb{P}\left(b_{n}=0\right.$ wins $\left.\mid b_{n}=b_{m}=0\right)<1$. Then bidding slightly above zero yields $\mathrm{MM} n \mathrm{H}$ strictly larger expected profit than $b_{n}=0$, $\bar{\Pi}_{n}(\epsilon \mid$ sell $)>\bar{\Pi}_{n}(0 \mid$ sell $)$, contradicting $u=0$. Next, suppose by way of contradiction that $u=1$. Then $\max _{b} \bar{\Pi}_{n}(b \mid$ sell $)=0$, for $n=1,2$. However, $\min \left\{p_{1}, p_{2}\right\} \in(0,1)$. Say $p_{m}<1$; then $l_{m} \leq \mathbb{E}[V]=\frac{1}{2}$. Therefore, $\bar{\Pi}_{n}\left(\frac{3}{4} \mid\right.$ sell $)=\frac{1}{4}\left(1-p_{m}\right)>0$, contradicting $\max _{b} \bar{\Pi}_{n}(b \mid$ sell $)=0$.

Step 4: $p_{n}=1 \Rightarrow l_{m}=0 ; \max \left\{p_{1}, p_{2}\right\}<1 \Rightarrow \max \left\{l_{1}, l_{2}\right\}<\mathbb{E}[V \mid$ sell $]$. The first part is trivial; we prove the second part. By Step 1, for both MMs and given any bid $b$, the probability of winning a sell order is maximized under $V=0$. Hence, for all $b$,

$$
\mathbb{E}\left[V \mid \text { sell, } b_{n}=b \text { wins }\right] \leq \mathbb{E}[V \mid \text { sell }]
$$

This implies, in turn, $l_{n} \leq \mathbb{E}[V \mid$ sell $]$, otherwise $\mathrm{MM} n \mathrm{U}$ could profitably deviate to $b_{n}=0$. Now suppose $\max \left\{p_{1}, p_{2}\right\}<1$ and, by way of contradiction, that $l_{m}=\mathbb{E}[V \mid$ sell $]$. We consider two cases, $u=\mathbb{E}[V \mid$ sell $]$ (Case 1) and $u>\mathbb{E}[V \mid$ sell $]$ (Case 2). In Case 1, Step 2 gives $u=\mathbb{E}[V \mid$ sell $] \in \bar{A}_{m}$. But then bidding slightly above $u$ yields $\mathrm{MM} n \mathrm{H}$ strictly larger expected profit than $b_{n}=u: \exists \varepsilon>0$ such that

$$
\bar{\Pi}_{n}(u+\epsilon \mid \text { sell })>\bar{\Pi}_{n}(u-x \mid \text { sell }), \quad \forall x \in[0, \varepsilon]
$$

contradicting $u=\sup \bar{\Sigma}_{n}$. Consider next Case 2. Note that in this case, by virtue of Steps 1 and 2 , there exists $\delta>0$ with

$$
\mathbb{P}\left(b_{m}=b \text { wins } \mid V=1\right)<\mathbb{P}\left(b_{m}=b \text { wins } \mid V=0\right)-\delta, \quad \forall b \leq \mathbb{E}[V \mid \text { sell }]
$$

Thus, $\exists \delta^{\prime}>0$ such that

$$
\mathbb{E}\left[V \mid \text { sell }, b_{m}=b \text { wins }\right]<\mathbb{E}[V \mid \text { sell }]-\delta^{\prime}, \quad \forall b \leq \mathbb{E}[V \mid \text { sell }]
$$

We therefore obtain

$\Pi_{m}(b \mid$ sell $)=\mathbb{P}\left(b_{m}=b\right.$ wins $)\left(\mathbb{E}\left[V \mid\right.\right.$ sell,$b_{m}=b$ wins $\left.]-b\right)<0, \quad \forall b \in\left[\mathbb{E}[V \mid\right.$ sell $]-\delta^{\prime}, \mathbb{E}[V \mid$ sell $\left.]\right]$ giving $l_{m} \leq \mathbb{E}[V \mid$ sell $]-\delta^{\prime}$. 
Step 5: $\left(A_{1} \cup \bar{A}_{1}\right) \cap\left(A_{2} \cup \bar{A}_{2}\right)=\emptyset$. That $\bar{A}_{n} \cap\left(A_{m} \cup \bar{A}_{m}\right)=\emptyset$ is trivial. Next, suppose by way of contradiction that we can find $b \in\left(A_{1} \cap A_{2}\right) \backslash\left(\bar{A}_{1} \cup \bar{A}_{2}\right)$. Then $b<\mathbb{E}[V \mid$ sell $]$, by virtue of Step 4 . Let $\Delta=\mathbb{E}[V \mid$ sell $]-b$, and consider $n$ such that $\mathbb{P}(\mathrm{MM} n$ wins $\mid$ tie at $b)<1$. Notice that $\Pi_{n}\left(b+\varepsilon \Delta \mid\right.$ sell,$\left.b_{m}=b\right)=\mathbb{E}[V \mid$ sell $]-b-\varepsilon \Delta$ as (i) given $b_{m}=b, b_{n}=b+\varepsilon \Delta$ always wins, and (ii) conditional on $b_{m}=b, \mathrm{MM} m$ is uninformed with probability 1 , from which $\mathbb{E}\left[V \mid\right.$ sell, $\left.b_{m}=b\right]=\mathbb{E}[V \mid$ sell $]$. Then,

$$
\begin{aligned}
& \Pi_{n}(b+\varepsilon \Delta \mid \text { sell })-\Pi_{n}(b \mid \text { sell }) \\
& =\mathbb{P}\left(b_{m}=b\right)\left(\Pi_{n}\left(b+\varepsilon \Delta \mid \text { sell, } b_{m}=b\right)-\Pi_{n}\left(b \mid \text { sell }, b_{m}=b\right)\right) \\
& +\left(1-\mathbb{P}\left(b_{m}=b\right)\right)\left(\Pi_{n}\left(b+\varepsilon \Delta \mid \text { sell }, b_{m} \neq b\right)-\Pi_{n}\left(b \mid \text { sell }, b_{m} \neq b\right)\right) \\
& =\mathbb{P}\left(b_{m}=b\right)(\mathbb{E}[V \mid \text { sell }]-b-\varepsilon \Delta-\mathbb{P}(\mathrm{MM} n \text { wins } \mid \text { tie at } b) \Delta) \\
& +\left(1-\mathbb{P}\left(b_{m}=b\right)\right)\left(\Pi_{n}\left(b+\varepsilon \Delta \mid \text { sell, } b_{m} \neq b\right)-\Pi_{n}\left(b \mid \text { sell, } b_{m} \neq b\right)\right) \\
& =\mathbb{P}\left(b_{m}=b\right)((1-\varepsilon) \Delta-\mathbb{P}(\mathrm{MM} n \text { wins } \mid \text { tie at } b) \Delta) \\
& +\left(1-\mathbb{P}\left(b_{m}=b\right)\right)\left(\Pi_{n}\left(b+\varepsilon \Delta \mid \text { sell }, b_{m} \neq b\right)-\Pi_{n}\left(b \mid \text { sell }, b_{m} \neq b\right)\right) .
\end{aligned}
$$

As $\lim _{\varepsilon \rightarrow 0}\left(\Pi_{n}\left(b+\varepsilon \Delta \mid\right.\right.$ sell,$\left.b_{m} \neq b\right)-\Pi_{n}\left(b \mid\right.$ sell,$\left.\left.b_{m} \neq b\right)\right)=0$, we obtain

$$
\lim _{\varepsilon \rightarrow 0}\left(\Pi_{n}(b+\varepsilon \Delta \mid \text { sell })-\Pi_{n}(b \mid \text { sell })\right)=(1-\mathbb{P}(\mathrm{MM} n \text { wins } \mid \text { tie at } b)) \Delta>0,
$$

contradicting $b \in A_{n}$.

Step 6: $\inf \left\{\Sigma_{1} \cup \bar{\Sigma}_{1}\right\}=\inf \left\{\Sigma_{2} \cup \bar{\Sigma}_{2}\right\}$. Assume $\max \left\{p_{1}, p_{2}\right\}<1$ (other cases are similar), so that, by Step 2, $\inf \left\{\Sigma_{1} \cup \bar{\Sigma}_{1}\right\}=\inf \Sigma_{1}$ and $\inf \left\{\Sigma_{2} \cup \bar{\Sigma}_{2}\right\}=\inf \Sigma_{2}$. Suppose by way of contradiction that $b=\inf \Sigma_{n}>\inf \Sigma_{m}=b^{\prime}$. Then $b \in\left(A_{m} \cup \bar{A}_{m}\right)$, otherwise we could find $\varepsilon>0$ such that

$$
\Pi_{n}(b-\varepsilon \mid \text { sell })>\Pi_{n}(b+x \mid \text { sell }), \quad \forall x \in[0, \varepsilon],
$$

contradicting $b=\inf \Sigma_{n}$, as then MMn could benefit from bidding below $b$. Applying Step 5 thus yields $b \notin\left(A_{n} \cup \bar{A}_{n}\right)$. Next, $b=\inf \Sigma_{n}$ together with $b \notin A_{n}$ implies $\sigma_{n}(b)=0$. Therefore, using Steps 1 and $2, b \in A_{m}$ would imply $\Pi_{m}(b \mid$ sell $)=-\gamma p_{n} b<0$, which cannot 
be. Similarly, $b \in \bar{A}_{m}$ would imply $\bar{\Pi}_{m}(b \mid$ sell $)=0$, which cannot be since, by virtue of Steps 2 and $3, \max _{b} \bar{\Pi}_{m}(b \mid$ sell $)>0$.

Step 7: $0 \in\left(\Sigma_{1} \cup \bar{\Sigma}_{1}\right) \cap\left(\Sigma_{2} \cup \bar{\Sigma}_{2}\right)$. Assume $\max \left\{p_{1}, p_{2}\right\}<1$ (other cases are similar). By Step $2, \inf \left\{\Sigma_{1} \cup \bar{\Sigma}_{1}\right\}=\inf \Sigma_{1}$ and $\inf \left\{\Sigma_{2} \cup \bar{\Sigma}_{2}\right\}=\inf \Sigma_{2}$. Let $b$ denote the common infinimum uncovered in Step 6, and suppose for a contradiction that $b>0$. By Step 5, one of the MMs does not have an atom at $b$. Consider $n$ such that $b \notin\left(A_{n} \cup \bar{A}_{n}\right)$. Then, by Step 1 ,

$$
\lim _{\varepsilon \rightarrow 0} \Pi_{m}(b+\varepsilon \mid \text { sell })=-\gamma p_{n} b<0
$$

contradicting $b \in \Sigma_{m}$.

Step 8: $\Sigma_{1} \cup \bar{\Sigma}_{1}=\Sigma_{2} \cup \bar{\Sigma}_{2}$. Suppose by way of contradiction that there exists $b^{\prime} \in\left(\Sigma_{n} \cup \bar{\Sigma}_{n}\right) \backslash$ $\left(\Sigma_{m} \cup \bar{\Sigma}_{m}\right)$, say $b^{\prime} \in \Sigma_{n} \backslash\left(\Sigma_{m} \cup \bar{\Sigma}_{m}\right)$ (the other case is similar). Then $b^{\prime} \in \arg \max _{b} \Pi_{n}(b \mid$ sell). Moreover, by Step $7, b^{\prime}>0$, and we can find $\delta>0$ such that $\left[b^{\prime}-\delta, b^{\prime}+\delta\right] \cap\left(\Sigma_{m} \cup \bar{\Sigma}_{m}\right)=$ $\emptyset$. Hence $\mathrm{MM} n$ can lower his bid at $b^{\prime}$ without decreasing his winning probability, giving $\Pi_{n}\left(b^{\prime}-\delta \mid\right.$ sell $)>\Pi_{n}\left(b^{\prime} \mid\right.$ sell $)=\max _{b} \Pi_{n}(b \mid$ sell $)$, which cannot be.

Step 9: $\Sigma_{1} \cup \bar{\Sigma}_{1}=\Sigma_{2} \cup \bar{\Sigma}_{2}=[0, u]$. By Steps 2, 3, 7 and 8 all that remains to be shown is that the common support is an interval. Suppose by way of contradiction that this is not the case. Then we can find $b^{\prime \prime}>b^{\prime}$, both in the common support, and such that $\left(b^{\prime}, b^{\prime \prime}\right) \cap\left(\Sigma_{1} \cup \bar{\Sigma}_{1}\right)=\emptyset$. By Step 5, there exists $n$ such that $b^{\prime \prime} \notin\left(A_{n} \cup \bar{A}_{n}\right)$. Hence, $\exists \varepsilon>0$ such that, $\forall x \in[0, \varepsilon]$, $\Pi_{m}\left(b^{\prime \prime}-\varepsilon \mid\right.$ sell $)>\Pi_{m}\left(b^{\prime \prime}+x \mid\right.$ sell $)$ and $\bar{\Pi}_{m}\left(b^{\prime \prime}-\varepsilon \mid\right.$ sell $)>\bar{\Pi}_{m}\left(b^{\prime \prime}+x \mid\right.$ sell $)$, contradicting $b^{\prime \prime} \in$ $\left(\Sigma_{m} \cup \bar{\Sigma}_{m}\right)$.

Step 10: $p_{n} \in(0,1) \Rightarrow l_{n}<u$. Suppose by way of contradiction that $p_{n} \in(0,1)$ and $l_{n}=u$. Then, by Step 2, $u \in \bar{A}_{n}$. Assume $\mathbb{P}(\mathrm{MM} m$ wins $\mid$ tie at $u)<1$ (the other case is similar). Then there exists $\varepsilon>0$ such that

$$
\bar{\Pi}_{m}(u+\epsilon \mid \text { sell })>\bar{\Pi}_{m}(u-x \mid \text { sell }), \quad \forall x \in[0, \varepsilon],
$$

contradicting $u \in \bar{\Sigma}_{m}$. 
Step 11: $A_{1} \cup \bar{A}_{1} \cup A_{2} \cup \bar{A}_{2} \subseteq\{0\}$. Suppose by way of contradiction that there exists $b \in$ $\left(A_{m} \cup \bar{A}_{m}\right)$, with $b>0$. Then, by virtue of Step $4, \exists \varepsilon>0$ such that, $\forall x \in(0, \varepsilon), \bar{\Pi}_{n}(b+$ $x \mid$ sell $)>\bar{\Pi}_{n}(b-x \mid$ sell $)$ and $\Pi_{n}(b+x \mid$ sell $)>\Pi_{n}(b-x \mid$ sell $)$. Thus $(b-\varepsilon, b) \cap\left(\Sigma_{n} \cup \bar{\Sigma}_{n}\right)=\emptyset$, contradicting Step 9.

Step 12: $\max _{b} \bar{\Pi}_{1}(b \mid$ sell $)=\max _{b} \bar{\Pi}_{2}(b \mid$ sell $)$. The combination of Steps 2, 3 and 11 shows that $\max _{b} \bar{\Pi}_{1}(b \mid$ sell $)=\bar{\Pi}_{1}(u \mid$ sell $)=(1-u)=\bar{\Pi}_{2}(u \mid$ sell $)=\max _{b} \bar{\Pi}_{2}(b \mid$ sell $)$.

Step 13: $0<p_{m}<p_{n}<1 \Rightarrow 0<l_{n}<l_{m}$. Let $0<p_{m}<p_{n}<1$ and suppose by way of contradiction that $l_{n} \geq l_{m}$. Note first that $l_{n}>0$, for otherwise $\{0\} \in A_{n} \cap A_{m}$, which Step 5 ruled out. Hence, by Step 11, neither MM has an atom at $l_{n}$. Steps 2 and 9 therefore yield $\max _{b} \bar{\Pi}_{n}(b \mid$ sell $)=\bar{\Pi}_{n}\left(l_{n} \mid\right.$ sell $)$ and $\max _{b} \bar{\Pi}_{m}(b \mid$ sell $)=\bar{\Pi}_{m}\left(l_{n} \mid\right.$ sell $)$. On the other hand, $\bar{\Pi}_{n}\left(l_{n} \mid\right.$ sell $) \geq\left(1-p_{m}\right)\left(1-l_{n}\right)$ and $\bar{\Pi}_{m}\left(l_{n} \mid\right.$ sell $)=\left(1-p_{n}\right)\left(1-l_{n}\right)$. As $p_{n}>p_{m}$, combining the previous remarks yields

$$
\max _{b} \bar{\Pi}_{n}(b \mid \text { sell })>\max _{b} \bar{\Pi}_{m}(b \mid \text { sell }),
$$

contradicting Step 12. Therefore, $l_{n}<l_{m}$. We next show that $l_{n}>0$. Suppose by way of contradiction that $l_{n}=0$. Then $0 \in A_{n}$, and, applying Step $5,0 \notin A_{m}$. We therefore obtain

$$
\Pi_{n}(0 \mid \text { sell })=\max _{b} \Pi_{n}(b \mid \text { sell })=0<\max _{b} \Pi_{m}(b \mid \text { sell }) .
$$

Yet, as $l_{m}>0$, Steps 9,11 and 12 give

$$
\begin{aligned}
\Pi_{n}\left(l_{m} \mid \text { sell }\right) & =-\gamma l_{m}+(1-\gamma) \bar{\Pi}_{n}\left(l_{m} \mid \text { sell }\right) \\
& =-\gamma l_{m}+(1-\gamma) \bar{\Pi}_{m}\left(l_{m} \mid \text { sell }\right) \\
& =\Pi_{m}\left(l_{m} \mid \text { sell }\right),
\end{aligned}
$$

contradicting $(\mathrm{C} 1)$.

Step 14: $p_{n}>p_{m} \Rightarrow 0=\Pi_{m}<\Pi_{n}<\bar{\Pi}_{n}=\bar{\Pi}_{m}$. Assume $0<p_{m}<p_{n}<1$ (other cases are similar). By Step 12, $\bar{\Pi}_{n}=\bar{\Pi}_{m}$. Moreover, Steps 1,2, 9, 11 and 13 give

$$
\Pi_{n}\left(l_{n} \mid \text { sell }\right)=-\gamma\left(p_{m}+\left(1-p_{m}\right) \sigma_{m}\left(l_{n}\right)\right) l_{n}+(1-\gamma) \bar{\Pi}_{n}\left(l_{n} \mid \text { sell }\right)<\bar{\Pi}_{n}\left(l_{n} \mid \text { sell }\right) .
$$

Hence $\Pi_{n}<\bar{\Pi}_{n}$ (by symmetry of the bid and ask sides of the market). We next show that 
$\Pi_{n}>\Pi_{m}$. Reasoning like we did above, and using Step 12 together with $l_{n}<l_{m}$,

$$
\begin{aligned}
\Pi_{n}\left(l_{n} \mid \text { sell }\right) & =-\gamma\left(p_{m}+\left(1-p_{m}\right) \sigma_{m}\left(l_{n}\right)\right) l_{n}+(1-\gamma) \bar{\Pi}_{n}\left(l_{n} \mid \text { sell }\right) \\
& >-\gamma l_{m}+(1-\gamma) \bar{\Pi}_{n}\left(l_{n} \mid \text { sell }\right) \\
& =-\gamma l_{m}+(1-\gamma) \bar{\Pi}_{m}\left(l_{m} \mid \text { sell }\right) \\
& =\Pi_{m}\left(l_{m} \mid \text { sell }\right)
\end{aligned}
$$

Hence, $\Pi_{n}>\Pi_{m}$. Lastly, we show that $\Pi_{m}=0$. Suppose by way of contradiction that $\Pi_{m}>0$. Then Steps 2 and 7 imply $0 \in A_{n}$. It ensues, using Step 5 , that $0 \notin\left(A_{m} \cup \bar{A}_{m}\right)$. We thus obtain $\Pi_{n}=0<\Pi_{m}$, contradicting $\Pi_{n}>\Pi_{m}$.

Proposition C1. For all $p_{1}, p_{2}$ and $q$, a trading equilibrium exists. Moreover, except for $p_{1}=p_{2}=q=0$ and $p_{1}=p_{2}=1$, any two trading equilibria induce the same strategies and differ at most by the tie-breaking rules they induce. ${ }^{31}$ For all $p_{1}, p_{2}$ and $q, \bar{\Pi}_{n}, \Pi_{n}$, $\bar{\Pi}_{S}$ and $\Pi_{S}$ are independent of the trading equilibrium considered.

Proof: The cases $p_{1}=p_{2}=0$ and $p_{1}=p_{2}=1$ are trivial. We prove below the existence of a trading equilibrium and the uniqueness of the strategies for $0<p_{m} \leq p_{n}<1$ and $q>0$ (other cases are similar).

We start by showing that in any trading equilibrium the speculator sells (resp. buys) with probability 1 when she is informed and $V=0$ (resp. $V=1$ ), and abstains when she is uninformed. First note that, by Lemma $\mathrm{C} 1, \hat{b}<1$ with probability 1 . So selling the asset is a strictly dominated strategy of the informed speculator when $V=1$. Similarly, buying the asset is a strictly dominated strategy of the informed speculator when $V=0$. Next, Suppose by way of contradiction that the speculator abstains with positive probability when she is informed and $V=0$ (the other case is similar, by symmetry). Then $\mathbb{P}(\hat{b}=0 \mid V=0)=1$, otherwise the speculator would have a profitable deviation. But then $\sigma_{1}(0)=\sigma_{2}(0)=1$, contradicting Step 5 in the proof of Lemma C1. We conclude that the speculator sells (resp. buys) with probability 1 when she is informed and $V=0$ (resp. $V=1$ ). We now prove that the speculator abstains when she is uninformed. Applying Lemma C1 gives $u<1$ and $\max \left\{l_{1}, l_{2}\right\}<\mathbb{E}[V \mid$ sell $]$. As we showed above that the speculator buys (resp. sells) with probability 1 when she is informed and $V=1$ (resp. $V=0$ ), we obtain $\mathbb{E}[V \mid$ sell $]<\frac{1}{2}$. The

\footnotetext{
${ }^{31}$ If $p_{1}=p_{2}=q=0$ then the uninformed speculator is indifferent between trading and abstaining. If $p_{1}=p_{2}=1$ then any type of the speculator is indifferent between trading and abstaining.
} 
uninformed speculator's expected profit from selling the asset is therefore bounded above by

$$
\begin{gathered}
\mathbb{P}(\text { sell order executed by an uninformed MM })\left(\max \left\{l_{1}, l_{2}\right\}-\frac{1}{2}\right) \\
+\mathbb{P}(\text { sell order executed by an informed } \mathrm{MM})(u-1)<0 .
\end{gathered}
$$

By symmetry, the uninformed speculator's expected profit from buying the asset is negative as well.

We next derive equilibrium strategies of the MMs. Since we saw above that in any trading equilibrium the speculator trades if and only if she is informed, we obtain $\gamma=\mathbb{P}(V=0 \mid$ sell $)=$ $\left(\frac{\pi q}{2}+\frac{1-\pi}{4}\right) /\left(\frac{\pi q}{2}+\frac{1-\pi}{2}\right)$ in any trading equilibrium. Now, by virtue of Lemma $\mathrm{C} 1$, if the pricing strategies $\sigma_{m}, \sigma_{n}, \underline{\sigma}_{m}, \underline{\sigma}_{n}, \bar{\sigma}_{m}$ and $\bar{\sigma}_{n}$ are in equilibrium then $\underline{\sigma}_{m}(0)=\underline{\sigma}_{n}(0)=1$ and there exist $0<l_{n} \leq l_{m}<u<1$ such that:

$$
\begin{gathered}
{\left[\left(1-p_{m}\right)+p_{m} \bar{\sigma}_{m}(x)\right](1-x)=1-u, \quad \forall x \in\left[l_{m}, u\right] ;} \\
{\left[\left(1-p_{n}\right)+p_{n} \bar{\sigma}_{n}(x)\right](1-x)=1-u, \quad \forall x \in\left[l_{m}, u\right] ;} \\
\bar{\sigma}_{m}\left(l_{m}\right)=0 ; \\
-\gamma l_{m}+(1-\gamma)(1-u)=0 ; \\
-\gamma x+(1-\gamma)\left[\left(1-p_{n}\right)+p_{n} \bar{\sigma}_{n}(x)\right](1-x)=0, \quad \forall x \in\left[l_{n}, l_{m}\right] ; \\
\left(1-p_{m}\right) \sigma_{m}(x)(1-x)=1-u, \quad \forall x \in\left[l_{n}, l_{m}\right] ; \\
\bar{\sigma}_{n}\left(l_{n}\right)=0 ; \quad \forall x \in\left[0, l_{n}\right] ; \\
=-\gamma\left[p_{m}+\left(1-p_{m}\right) \sigma_{m}(x)\right] x+(1-\gamma)\left(1-p_{m}\right) \sigma_{m}(x)(1-x) \\
\left.\left.-\gamma\left[p_{n}+\left(1-p_{m}\right) \sigma_{m}\left(l_{n}\right)\right] l_{n}+(1-\gamma)\left(1-p_{m}\right) \sigma_{m}\left(l_{n}\right)\left(1-l_{n}\right), \quad \forall x\right)\right] x+(1-\gamma)\left(1-p_{n}\right) \sigma_{n}(x)(1-x)=0, \quad \forall x \in\left[0, l_{n}\right] .
\end{gathered}
$$

Equations (C2) and (C3) are the equiprofit conditions of, respectively, $\mathrm{MM} n \mathrm{H}$ and $\mathrm{MM} m \mathrm{H}$ in the bid range $\left[l_{m}, u\right]$; (C4) is obtained by definition of $l_{m}$; equation (C5) captures $\Pi_{m}\left(l_{m} \mid\right.$ sell $)=$ 0 ; equations $(\mathrm{C} 6)$ and $(\mathrm{C} 7)$ are the equiprofit conditions of, respectively, $\mathrm{MM} m \mathrm{U}$ and $\mathrm{MM} n \mathrm{H}$ in the bid range $\left[l_{n}, l_{m}\right] ;(\mathrm{C} 8)$ is obtained by definition of $l_{n}$; lastly, equations (C9) and (C10) are the equiprofit conditions of, respectively, $\mathrm{MM} n \mathrm{U}$ and $\mathrm{MM} m \mathrm{U}$ in the bid range $\left[0, l_{n}\right]$. That 
the system of equations (C2)-(C10) uniquely determines pricing strategies $\sigma_{m}, \sigma_{n}, \bar{\sigma}_{m}$ and $\bar{\sigma}_{n}$ is straightforward to check. ${ }^{32}$

By construction the strategies above are in equilibrium if no MM can profitably bid outside the support of their respective strategies. Observe to begin with that no MM can profitably bid outside $[0, u]$. So we only need to check the remaining cases. To see that $\mathrm{MM} m \mathrm{U}$ has no profitable deviation to $b \in\left(l_{m}, u\right]$ note that

$$
\Pi_{m}(b \mid \text { sell })=-\gamma b+(1-\gamma)\left[\left(1-p_{n}\right)+p_{n} \bar{\sigma}_{n}(b)\right](1-b), \quad \forall b \in\left[l_{m}, u\right] .
$$

Hence, by (C3),

$$
\Pi_{m}(b \mid \text { sell })=-\gamma b+(1-\gamma)(1-u), \quad \forall b \in\left[l_{m}, u\right] .
$$

The last highlighted equation gives $\Pi_{m}(b \mid$ sell $)<\Pi_{m}\left(l_{m} \mid\right.$ sell $)$, for all $b \in\left(l_{m}, u\right]$. Similarly, to see that $\mathrm{MM} m \mathrm{H}$ has no profitable deviation to $b \in\left[l_{n}, l_{m}\right)$ note that, by (C6),

$$
\bar{\Pi}_{m}(b \mid \text { sell })=\left[\left(1-p_{n}\right)+p_{n} \bar{\sigma}_{n}(b)\right](1-b)=\frac{\gamma b}{1-\gamma}, \quad \forall b \in\left[l_{n}, l_{m}\right]
$$

Hence $\bar{\Pi}_{m}(b \mid$ sell $)<\bar{\Pi}_{m}\left(l_{m} \mid\right.$ sell $)$ for all $b \in\left[l_{n}, l_{m}\right)$. MM $m$ H has no profitable deviation to $b \in\left[0, l_{n}\right]$ either, since, by $(\mathrm{C} 10)$,

$$
\bar{\Pi}_{m}(b \mid \text { sell })=\left(1-p_{n}\right) \sigma_{n}(b)(1-b)=\frac{\gamma\left[p_{n}+\left(1-p_{n}\right) \sigma_{n}(b)\right] b}{1-\gamma}, \quad \forall b \in\left[0, l_{n}\right] .
$$

Therefore, $\bar{\Pi}_{m}(b \mid$ sell $) \leq \bar{\Pi}_{m}\left(l_{n} \mid\right.$ sell $)$ for all $b \in\left[0, l_{n}\right]$, which, combined with the previous remark, gives $\bar{\Pi}_{m}(b \mid$ sell $)<\bar{\Pi}_{m}\left(l_{m} \mid\right.$ sell $)$ for all $b \in\left[0, l_{n}\right]$. This finishes to show that neither $\mathrm{MM} m \mathrm{U}$ nor $\mathrm{MM} m \mathrm{H}$ can profitably bid outside the support of their respective strategies. Similar arguments establish that neither $\mathrm{MM} n \mathrm{U}$ nor $\mathrm{MM} n \mathrm{H}$ can profitably bid outside the support of their respective strategies.

Lastly, Step 5 in the proof of Lemma C1 shows that for a tie to occur with positive probability requires both MMs to be informed, and either $V=0$ and a sell order or $V=1$ and a buy order. So MMs' profits are zero conditional on a tie, irrespective of the tie-breaking rule. It follows that uniqueness of the strategies implies uniqueness of $\bar{\Pi}_{n}, \Pi_{n}, \bar{\Pi}_{S}$ and $\Pi_{S}$.

\footnotetext{
${ }^{32}$ Combining $(\mathrm{C} 2)$ and $(\mathrm{C} 4)$ pins down $l_{m}$ in terms of $u$; (C5) then gives $u$ and, therefore, $l_{m}$ as well. Applying (C2) and (C3) now gives $\bar{\sigma}_{m}$ and $\bar{\sigma}_{n}$ over the interval $\left[l_{m}, u\right]$. Next, Combining $(\mathrm{C} 6)$ and $(\mathrm{C} 8)$ pins down $l_{n}$, while $(\mathrm{C} 6)$ and $(\mathrm{C} 7)$ then give $\bar{\sigma}_{n}$ and $\sigma_{m}$ over the interval $\left[l_{n}, l_{m}\right]$. Finally $(\mathrm{C} 9)$ and $(\mathrm{C} 10)$ give $\sigma_{m}$ and $\sigma_{n}$ over the interval $\left[l_{n}, l_{m}\right]$.
} 
The following technical lemma is used in the proof of Theorem 2.

Lemma C2. Let $p^{*}(c, \pi)$ be given by (A16) and

$$
H(c ; \pi):=\bar{\Pi}_{S}\left(p^{*}(c, \pi), 0\right)-c .
$$

Then $H(c ; \pi)=0$ has exactly one solution in the interval $c \in\left(0, \frac{1-\pi}{4}\right)$.

Proof: Consider any equilibrium of the trading game with $q=0$ and a given, arbitrary, $p$. Define $\beta:=\mathbb{E}\left[b_{n} \mid \mathrm{MM} n\right.$ is uninformed $]$ and $\hat{\beta}:=\mathbb{E}\left[b_{n} \mid\right.$ both MMs uninformed, $\left.b_{n} \geq b_{m}\right]$. By symmetry of the bid and ask sides of the market, we can write

$$
\bar{\Pi}_{S}(p, 0)=2 p(1-p) \beta+(1-p)^{2} \hat{\beta}
$$

and

$$
\Pi_{n}(p, 0)=\left(\frac{1-\pi}{2}\right)\left[\frac{1}{2}\left(p(0-\beta)+(1-p) \frac{1}{2}(0-\hat{\beta})\right)+\frac{1}{2}(1-p) \frac{1}{2}(1-\hat{\beta})\right] .
$$

Rearranging the last highlighted equation gives

$$
\begin{aligned}
\Pi_{n}(p, 0) & =\frac{1}{4}(1-p)-\frac{1}{2}[p \beta+(1-p) \hat{\beta}] \\
& =\frac{1}{4}(1-p)-\frac{1}{2} \frac{\bar{\Pi}_{S}(p, 0)}{1-p}+\frac{1}{2} p \beta .
\end{aligned}
$$

Hence, as $\Pi_{n}(p, 0)=0$,

$$
\bar{\Pi}_{S}(p, 0)=\frac{1}{2}(1-p)^{2}+p(1-p) \beta .
$$

Next, using (A9) gives

$$
\beta=\int b \sigma^{\prime}(b) d b=\frac{p\left(\frac{1}{1-2 b}+\ln \left(\frac{1}{2}-b\right)\right)}{4(1-p)} .
$$

Finally, combining (A16), (C13) and (C14) yields, for all $c \in\left(0, \frac{1-\pi}{4}\right)$,

$$
H(c ; \pi)=\frac{1}{(1-\pi-2 c)^{2}}\left[\frac{(1-\pi-4 c)^{2}}{4} \ln \left(1-\frac{4 c}{1-\pi}\right)+c(1-\pi-2 c)(2 c+\pi)\right] .
$$

Let $G(c ; \pi)$ denote the expression inside the square bracket. One verifies that: 
(i) $G(0 ; \pi)=0$;

(ii) $G(c ; \pi) \rightarrow \frac{(1-\pi)^{2}(1+\pi)}{16}$ as $c \rightarrow \frac{1-\pi}{4}$;

(iii) $G^{\prime}(0 ; \pi)<0<G^{\prime \prime}(0 ; \pi)$;

(iv) $G^{\prime \prime \prime}(c ; \pi)<0$ for all $c \in\left(0, \frac{1-\pi}{4}\right)$.

Therefore, $G(c ; \pi)=0$ has exactly one solution in the interval $c \in\left(0, \frac{1-\pi}{4}\right) .{ }^{33}$

\footnotetext{
${ }^{33}$ On the interval $\left(0, \frac{1-\pi}{4}\right)$, the function $G$ is first convex, then concave. The function starts below the horizontal axis, and ends above it. Suppose it crossed the horizontal axis twice. Then at the second crossing, the function has to be decreasing and concave. But this contradicts $G$ ending above the horizontal axis.
} 


\section{Appendix D: Trading Game with Observable Quotes (for online publication)}

In this appendix we analyze the trading game induced by the observable quotes model, with $z>0$ denoting the probability with which the speculator gets to observe the quotes before placing her market order. Specifically, throughout this appendix $p$ and $q$ play the role of parameters: each MM acquires information with probability $p$, while the speculator acquires information with probability $q$. A strategy of $\mathrm{MM} n$ comprises cumulative distribution functions $\sigma_{n}, \underline{\sigma}_{n}$ and $\bar{\sigma}_{n}$ specifying respectively the distribution of the bid price $b_{n}$ of $\mathrm{MM} n \mathrm{U}$, $\mathrm{MM} n \mathrm{~L}$ and $\mathrm{MM} n \mathrm{H}$. As the bid and ask sides of the market are symmetric we assume as usual that, conditional on $\mathrm{MM} n \mathrm{U}, 1-a_{n}$ is distributed like $b_{n}$. Similarly, we assume that the law of $1-a_{n}$ conditional on $\mathrm{MM} n \mathrm{~L}$ (resp. $\mathrm{MM} n \mathrm{H}$ ) is the same as the law of $b_{n}$ conditional on $\mathrm{MM} n \mathrm{H}$ (resp. $\mathrm{MM} n \mathrm{~L}$ ). A strategy of the speculator specifies her market order as a function of the information she possesses at that point. A WELM trading equilibrium is a perfect Bayesian equilibrium such that

(i) $\sigma_{1}=\sigma_{2}=\sigma, \underline{\sigma}_{1}=\underline{\sigma}_{2}=\underline{\sigma}$ and $\bar{\sigma}_{1}=\bar{\sigma}_{2}=\bar{\sigma}$;

(ii) $\underline{\sigma}(0)=1$;

(iii) either $p \in\{0,1\}$ or $\sigma$ and $\bar{\sigma}$ are atomless, with $\operatorname{supp}(\sigma)=[0, l]$ and $\operatorname{supp}(\bar{\sigma})=[l, u]$.

We focus throughout this appendix on $p \in(0,1)$ and $q<1$. The case $p=0$ is almost identical. If $q=1$, the observability of the quotes is inconsequential. The case $p=1$ is straightforward: both MMs set prices equal to the realized asset value. Lastly, to shorten the exposition, we introduce the indicator variables $I_{S}, I_{n}$ and $Z$ respectively equal to 1 if and only if (a) the speculator acquires information, (b) MMn acquires information, (c) quotes are observable.

Proposition D1. Assume $p \in(0,1)$ and $q<1$. In any WELM trading equilibrium:

$$
\begin{array}{rlrl}
\bar{\Pi}_{n}(p, q) & =\left(\frac{(1-\pi)(1-p)}{2}\right) \frac{1-\pi(1-2 q)+2 \pi p(1-q) z}{2-p-2 \pi(1-q)+\pi p(1+2(1-q) z)} ; \\
\sigma(b) & =\frac{(1+\pi(2 z-1)+2 \pi q(1-z)) p b}{(1-p)(1-\pi-2 b(1-\pi(1-q)))}, \quad \forall b \in[0, l] ; \\
\bar{\sigma}(b) & =\frac{2 \bar{\Pi}_{n}(p, q)-(1-p)(1-\pi)(1-b)}{(1-b)(1-\pi) p}, & \forall b \in[l, u] ;
\end{array}
$$




$$
\begin{gathered}
l=\frac{(1-\pi)(1-p)}{2-p-2 \pi(1-q)+\pi p(1+2(1-q) z)} ; \\
u=\frac{1-\pi-2 \bar{\Pi}_{n}(p, q)}{1-\pi} .
\end{gathered}
$$

In particular, $\bar{\Pi}_{n}(p, q), l$ and $u$ given by, respectively, (D1), (D4) and (D5) satisfy $\bar{\Pi}_{n}(p, q)>0$ and $0<l<u<1$.

Proof: We start with a few preliminary remarks. Observe that WELM equilibria are separating equilibria. Hence, in any WELM equilibrium, $I_{S} \vee\left(\left(I_{1} \vee I_{2}\right) \wedge Z\right)=1$ implies that, on the equilibrium path, the speculator learns the realization of $V$. In this case, by sequential rationality, the speculator buys if $V=1$ and sells if $V=0 .{ }^{34}$ On the equilibrium path sell orders are thus more likely conditional on $V=0$ than they are conditional on $V=1$, implying $l<\frac{1}{2}$. ${ }^{35}$ Hence, on the equilibrium path, the speculator abstains whenever $I_{S} \vee\left(\left(I_{1} \vee I_{2}\right) \wedge Z\right)=0 .{ }^{36}$

Next, by definition of a WELM trading equilibrium, MM $n$ U's expected profit on the bid side of the market has to be zero (the same being true of course on the ask side of the market). ${ }^{37}$ As $\mathrm{MM} n \mathrm{U}$ randomizes over $[0, l]$, we obtain

$$
\begin{gathered}
-\frac{1}{2}\left[\pi((1-p) q \sigma(b)+p(z+(1-z) q))+\left(\frac{1-\pi}{2}\right)(p+(1-p) \sigma(b))\right] b \\
+\frac{1}{2}\left(\frac{1-\pi}{2}\right)(1-p) \sigma(b)(1-b)=0, \quad \forall b \in[0, l] .
\end{gathered}
$$

The first term in equation (D6) can be decomposed as follows. With probability $\frac{1}{2}$ the asset value is $V=0$, in which case a winning bid $b$ induces a loss equal to $b$. With probability $\pi$ the trader is a speculator. By the remarks made earlier in this proof the speculator sells if and only if one of the following 3 cases occurs: (i) $I_{m}=0$ and $I_{S}=1$, (ii) $I_{m}=1$ and $Z=1$, (iii) $I_{m}=1, Z=0$ and $I_{S}=1$. In case (i) $\mathrm{MM} n \mathrm{U}$ has the winning bid with probability $\sigma(b)$; in cases (ii) and (iii) $\mathrm{MM} n \mathrm{U}$ has the winning bid with probability 1 . With probability $\frac{1-\pi}{2}$ the

\footnotetext{
${ }^{34}$ We suppose here, without loss of generality, that the speculators always trades when she is indifferent between trading and abstaining.

${ }^{35} \mathrm{MM} n \mathrm{U}$ is subject to greater adverse selection than in the baseline case. As $l<\frac{1}{2}$ in the baseline model, $l<\frac{1}{2}$ with observable quotes as well.

${ }^{36}$ Observe that on the equilibrium path, if $I_{S}=I_{1}=I_{2}=0$ and $Z=1$ then the speculator's expected profit from trading the asset (either buying or selling) is at most $l-\frac{1}{2}<0$. If instead $I_{S}=Z=0$ then her expected profit from trading the asset is bounded above by $\mathbb{P}$ (trade with an uninformed $\mathrm{MM} \mid I_{S} \vee Z=$ $0)\left(l-\frac{1}{2}\right)+\mathbb{P}\left(\right.$ trade with an informed $\left.\mathrm{MM} \mid I_{S} \vee Z=0\right)(u-1)<0$.

${ }^{37}$ This must be since $\mathrm{MM} n \mathrm{U}$ is indifferent between bids on the interval $[0, l]$, and the expected profit of $b_{n}=0$ is zero due to the remark that, in any WELM trading equilibrium, $\mathbb{P}\left(b_{n}=0\right.$ wins $\left.\mid V=1\right)=0$.
} 
trader is hit by the liquidity shock and sells the asset: either $I_{m}=1$, in which case $\mathrm{MM} n \mathrm{U}$ has the winning bid with probability 1 , or $I_{m}=0$, in which case $\mathrm{MM} n \mathrm{U}$ has the winning bid with probability $\sigma(b)$. The second term in equation (D6) is decomposed as follows. With probability $\frac{1}{2}$ the asset value is $V=1$, in which case a winning bid $b$ induces a gain equal to $1-b$. The probability of a sell order is the probability of a liquidity trader selling the asset, that is, $\frac{1-\pi}{2}$. Either $I_{m}=1$, in which case $\mathrm{MM} n \mathrm{U}$ has the losing bid, or $I_{m}=0$, in which case $\mathrm{MM} n \mathrm{U}$ has the winning bid with probability $\sigma(b)$.

As $\mathrm{MM} n \mathrm{H}$ randomizes over $[l, u]$ we obtain in a similar way

$$
\left(\frac{1-\pi}{2}\right)[p \bar{\sigma}(b)+(1-p)](1-b)=\left(\frac{1-\pi}{2}\right)(1-p)(1-l), \quad \forall b \in[l, u] .
$$

We can now conclude the proof of the proposition. Rearranging (D6) yields (D2), from which solving $\sigma(l)=1$ gives us (D4). Substituting (D4) into the right-hand side of (D7) and using the symmetry of the problem to write the resulting expression as $\bar{\Pi}_{n}(p, q)$ gives us (D1). Rearranging the terms in (D7) then yields (D3), from which solving $\bar{\sigma}(u)=1$ yields (D5). To see that $l>0$, substitute $b=l$ into (D6). Substituting $b=u$ into (D7) and using the fact that $l<\frac{1}{2}$ yields $u<1$ and $\bar{\Pi}_{n}(p, q)>0$.

Lemma D1. Assume $p \in(0,1)$ and $q<1$. Let $\sigma(\cdot), \bar{\sigma}(\cdot), l$ and $u$ be defined by (D2), (D3), (D4) and (D5), respectively. Then

$$
\arg \max _{b \in[0,1]}\left(\frac{1-\pi}{2}\right)[p \bar{\sigma}(b)+(1-p) \sigma(b)](1-b)=[l, u]
$$

and

$$
\begin{gathered}
\arg \max _{b \in[0,1]}-\frac{1}{2}\left[\pi((1-p) q \sigma(b)+p(z+(1-z) q))+\left(\frac{1-\pi}{2}\right)(p+(1-p) \sigma(b))\right] b \\
+\frac{1}{2}\left(\frac{1-\pi}{2}\right)[p \bar{\sigma}(b)+(1-p) \sigma(b)](1-b)=[0, l] .
\end{gathered}
$$

The maximum values of (D8) and (D9) are $\bar{\Pi}_{n}(p, q)$, given by (D1), and 0 , respectively.

Proof: By virtue of (D7),

$$
\left(\frac{1-\pi}{2}\right)[p \bar{\sigma}(b)+(1-p) \sigma(b)](1-b)=\left(\frac{1-\pi}{2}\right)(1-u), \quad \forall b \in[l, u] .
$$


As $\bar{\sigma}(u)=\sigma(u)=1$, notice that the left-hand side of (D10) is strictly decreasing in $b$ for $b \geq u$. Next, rewriting (D6) as

$$
\begin{aligned}
&-\frac{1}{2}\left[\pi((1-p) q \sigma(b)+p(z+(1-z) q))+\left(\frac{1-\pi}{2}\right)(p+(1-p) \sigma(b))\right] b \\
&+\frac{1}{2}\left(\frac{1-\pi}{2}\right)[p \bar{\sigma}(b)+(1-p) \sigma(b)](1-b)=0, \quad \forall b \in[0, l],
\end{aligned}
$$

gives

$$
\left(\frac{1-\pi}{2}\right)[p \bar{\sigma}(b)+(1-p) \sigma(b)](1-b)=\left[\pi((1-p) q \sigma(b)+p(z+(1-z) q))+\frac{1-\pi}{2}(p+(1-p) \sigma(b))\right] b,
$$

for all $b \in[0, l]$. The right-hand side of the last highlighted equation is strictly increasing in $b$. So combining the previous steps yields (D8). Finally, (D8) and the observation that the first term in the maximand of (D9) is a strictly decreasing function of $b$ together yield (D9).

Proposition D2. Assume $p \in(0,1)$ and $q<1$. Let $\sigma(\cdot), \bar{\sigma}(\cdot), l$ and $u$ be defined by (D2), (D3), (D4) and (D5), respectively. Define

$$
h(b):=\frac{(1-u-b)(1-\pi)-2 b \pi q}{2 b \pi(1-q) z p},
$$

and suppose that

$$
1-\bar{\sigma}(b) \geq h(b), \quad \forall b \in[l, u] .
$$

Then a WELM trading equilibrium exists.

Proof: The following notation will be used throughout the proof. Let the cdfs $\sigma$ and $\bar{\sigma}$ be defined by (D2) and (D3), respectively. Define also the $\operatorname{cdf} \underline{\sigma}$ such that $\underline{\sigma}(0)=1$. Let $\Gamma$ denote the set of bid-ask price pairs $\left(b_{n}, a_{n}\right)$ consistent with the strategies $\sigma, \underline{\sigma}$, and $\bar{\sigma}$, that is,

$$
\Gamma=(\{0\} \times[1-u, 1-l]) \cup([0, l] \times[1-l, 1]) \cup([l, u] \times\{1\}) .
$$

Similarly, let $\Gamma^{+}$denote the set of tuples $\left(b_{1}, a_{1}, b_{2}, a_{2}\right)$ consistent with the strategies $\sigma, \underline{\sigma}$, and 
$\bar{\sigma}$, that is,

$$
\begin{aligned}
\Gamma^{+}=\left\{\left(b_{1}, a_{1}, b_{2}, a_{2}\right):\right. & \left(b_{1}, a_{1}\right) \in \Gamma,\left(b_{2}, a_{2}\right) \in \Gamma, \\
\left(b_{n}, a_{n}\right) & \in[l, u] \times\{1\} \Rightarrow\left(b_{m}, a_{m}\right) \notin\{0\} \times[1-u, 1-l], \\
\left(b_{n}, a_{n}\right) & \left.\in\{0\} \times[1-u, 1-l] \Rightarrow\left(b_{m}, a_{m}\right) \notin[l, u] \times\{1\}\right\} .
\end{aligned}
$$

Let $\beta: \Gamma^{+} \rightarrow\left\{0, \frac{1}{2}, 1\right\}$ represent the mapping from consistent tuples $\left(b_{1}, a_{1}, b_{2}, a_{2}\right)$ to posterior beliefs that $V=1$, computed through Bayes' rule. Let $\mu_{n}$ denote the speculator's belief that $V=1$ based only on the quotes of $\mathrm{MM} n$, with $\mu_{n}=\emptyset$ in case $\left(b_{n}, a_{n}\right) \notin \Gamma{ }^{38}$ Let $\mu$ denote the speculator's belief that $V=1$ at the time she chooses her market order.

Assume the condition (C) holds with equality (the other case is similar). We aim to show that the following strategies, beliefs and tie-breaking rule comprise a trading equilibrium:

(I) $\sigma_{1}=\sigma_{2}=\sigma$;

(II) $\underline{\sigma}_{1}=\underline{\sigma}_{2}=\underline{\sigma}$;

(III) $\bar{\sigma}_{1}=\bar{\sigma}_{2}=\bar{\sigma}$

(IV) if $I_{S}=1$ then $\mu=v$;

$(\mathrm{V})$ if $I_{S} \vee Z=0$ then $\mu=\frac{1}{2}$;

(VI) if $I_{S}=0$ and $Z=1$ then:

$$
\mu= \begin{cases}\beta\left(b_{1}, a_{1}, b_{2}, a_{2}\right) & \text { if }\left(b_{1}, a_{1}, b_{2}, a_{2}\right) \in \Gamma^{+} ; \\ I_{\left\{1-a_{m}>b_{n}\right\}} & \text { if } \mu_{n}=1 \text { and } \mu_{m}=0 ; \\ \mu_{m} & \text { if } \mu_{m} \in\{0,1\} \text { and } \mu_{n}=\emptyset ; \\ \frac{a_{n}+b_{n}}{2} & \text { if } \mu_{m}=\frac{1}{2}, \mu_{n}=\emptyset, \text { and } b_{n}<a_{n} ; \\ 1 & \text { if } \mu_{m}=\frac{1}{2}, a_{n} \leq b_{n}, \text { and } a_{n} \neq \hat{a} ; \\ 0 & \text { if } \mu_{m}=\frac{1}{2}, a_{n} \leq b_{n}, a_{n}=\hat{a} \text { but } b_{n} \neq \hat{b} ; \\ \frac{a_{n}+b_{n}}{2} & \text { if } \mu_{m}=\frac{1}{2}, a_{n} \leq b_{n}, a_{n}=\hat{a} \text { and } b_{n}=\hat{b} .\end{cases}
$$

\footnotetext{
${ }^{38}$ We use the terminology "speculator's belief that $V=1$ " for the probability which the speculator attaches to the event $V=1$.
} 
(VII) ties are broken uniformly at random, except if $\mu_{m}=\frac{1}{2}, a_{n} \leq b_{n}, a_{n}=\hat{a}$ and $b_{n}=\hat{b}$, in which case any tie is broken in favor of $\mathrm{MM} n$;

(VIII) the speculator's market order satisfies sequential rationality with the additional requirement that if $I_{S}=0, Z=1, \mu_{m}=\frac{1}{2}, a_{n} \leq b_{n}, a_{n}=\hat{a}$ and $b_{n}=\hat{b}$ (in which case, by (D12g), $\mu=\frac{a_{n}+b_{n}}{2}$ ) then the speculator buys with probability $\frac{1}{2}$ and sells with probability $\frac{1}{2}$.

The proposed equilibrium has the following features. If the speculator acquires information her beliefs concerning $V$ are determined by the realized value $v$, that is, even if the quotes suggest otherwise (see (IV)). If the speculator does not acquire information and quotes are unobservable then $\mu$ is equal to the prior belief that $V=1$, that is, $\mu=\frac{1}{2}$ (see $(\mathrm{V})$ ). The case in which the speculator does not acquire information but gets to observe the quotes is subdivided into 7 cases. If the quotes are consistent with the proposed equilibrium strategies, then $\mu$ is derived using Bayes' rule (see (D12a)). If MMn's quotes signals $V=1$ while MMm's quotes signals $V=0$, that is, $\left(b_{n}, a_{n}\right) \in[l, u] \times\{1\}$ and $\left(b_{m}, a_{m}\right) \in\{0\} \times[1-u, 1-l]$, then $\mu=1$ if $1-a_{m}>b_{n}$ and $\mu=0$ otherwise (see (D12b)). If MMn's quotes are inconsistent with the proposed equilibrium strategies but MMm's quotes signal that $\mathrm{MM} m$ is informed then the speculator ignores $\mathrm{MM} n$ and bases her beliefs exclusively on the quotes of $\mathrm{MM} m$ (see (D12c)). The case in which MMn's quotes are inconsistent with the proposed equilibrium strategies and MMm's quotes signal that $\mathrm{MM} m$ is uninformed are further subdivided into 4 cases. If MMn's quotes satisfy $b_{n}<a_{n}$ then $\mu=\frac{a_{n}+b_{n}}{2}$ (see (D12d)), in which case sequential rationality precludes trading between the speculator and MMn. If $a_{n} \leq b_{n}$ and MMn does not offer the best ask price then $\mu=1$, (see (D12e)), in which case sequential rationality precludes trading between the speculator and $\mathrm{MM} n{ }^{39}$ If $a_{n} \leq b_{n}, \mathrm{MM} n$ offers the best ask price but not the best bid price then $\mu=0$, (see (D12f)), in which case sequential rationality precludes trading between the speculator and MMn. Lastly, if $a_{n} \leq b_{n}$ and MMn offers the best bid and ask prices then $\mu=\frac{a_{n}+b_{n}}{2}$ (see (D12g)), in which case the tie-breaking rule ensures that, conditional on placing a market order, the speculator trades with MMn (see (VII)).

Note that the proposed equilibrium satisfies the requirements of a WELM equilibrium; thus, repeating arguments used to prove Proposition D1, on the equilibrium path, the specu-

\footnotetext{
${ }^{39}$ Observe that $a_{n} \neq \hat{a}$ implies $\hat{a}<1$. So, for $\mu=1$, the speculator's expected profit from buying the asset is strictly positive. On the other hand, the speculator's expected profit from selling is at most 0 . Sequential rationality therefore requires the speculator to buy.
} 
lator learns the realization of $V$ if $I_{S} \vee\left(\left(I_{1} \vee I_{2}\right) \wedge Z\right)=1$. Moreover, since $l<\frac{1}{2}$, sequential rationality requires the speculator to abstain if $I_{S} \vee\left(\left(I_{1} \vee I_{2}\right) \wedge Z\right)=0 .{ }^{40}$ It ensues that, on the equilibrium path, MM $n \mathrm{U}$ 's expected profit on the bid side of the market can be written as the left-hand side of (D6). Similarly, on the equilibrium path, MMnH's expected profit on the bid side of the market can be written as the left-hand side of (D7). These remarks, Lemma D1 and the symmetry of the bid and ask sides of the market together establish that, on the equilibrium path: $\mathrm{MM} n \mathrm{U}$ 's expected profit is equal to 0, while MM $n \mathrm{H}$ 's expected profit equals $\bar{\Pi}_{n}(p, q)$ given by (D1). We establish in the rest of the proof that neither $\mathrm{MM} n \mathrm{U}$ nor $\mathrm{MM} n \mathrm{H}$ have a profitable deviation (which, by symmetry, implies that $\mathrm{MM} n \mathrm{~L}$ does not have a profitable deviation either).

Step 1: there exists no profitable deviation of MMnU to $\left(a_{n}, b_{n}\right) \notin \Gamma$, with $b_{n}<a_{n}$.

Suppose $\mathrm{MM} n \mathrm{U}$ deviates to $\left(\tilde{a}_{n}, \tilde{b}_{n}\right) \notin \Gamma$, with $\tilde{b}_{n}<\tilde{a}_{n}$. Observe first that in this case, applying (IV), (V), (D12c) and (D12d), the speculator trades with $\mathrm{MM} n \mathrm{U}$ if and only if $I_{S} \vee\left(I_{m} \wedge Z\right)=1$ (notice that if $I_{S}=I_{m}=0$ while $Z=1$ then (D12d) yields $b_{n}<\mu<a_{n}$ ). So the "demand" facing $\mathrm{MM} n \mathrm{U}$ is the same as it is on the equilibrium path. In consequence, $\mathrm{MM} n \mathrm{U}$ 's expected profit on the bid side of the market can be written like the maximand of (D9), with $b=\tilde{b}_{n}$. Yet, by virtue of Lemma D1, the maximand of (D9) is maximized when $\mathrm{MM} n \mathrm{U}$ sticks to the proposed equilibrium strategy. The symmetry between the bid and ask sides of the market finishes to establish that $\left(\tilde{a}_{n}, \tilde{b}_{n}\right)$ is not a profitable deviation of $\mathrm{MM} n \mathrm{U}$.

\footnotetext{
${ }^{40}$ See the third footnote in the proof of Proposition D1.
} 
Step 2: there exists no profitable deviation of $\mathrm{MM} n \mathrm{U}$ to $\left(a_{n}, b_{n}\right) \notin \Gamma$, with $a_{n} \leq b_{n}$.

Suppose $\mathrm{MM} n \mathrm{U}$ deviates to $\left(\tilde{a}_{n}, \tilde{b}_{n}\right) \notin \Gamma$, with $\tilde{a}_{n} \leq \tilde{b}_{n}$. Now in this case, applying (IV), (V), (D12c), (D12e), (D12f), (D12g), (VII) and (VIII) the speculator trades with MMn if and only if either (a) $I_{S} \vee\left(I_{m} \wedge Z\right)=1$ or (b) $I_{S} \vee I_{m}=0, Z=1, \tilde{a}_{n}=\hat{a}$ and $\tilde{b}_{n}=\hat{b}$. Moreover, in the latter event, (VIII) assures that the speculator buys with probability $\frac{1}{2}$ and sells with probability $\frac{1}{2}$. These remarks enable us to write the expected profit of $\mathrm{MM} n \mathrm{U}$ as

$$
\begin{gathered}
\left\{-\frac{1}{2}\left[\pi\left((1-p) q \sigma\left(\tilde{b}_{n}\right)+p(z+(1-z) q)\right)+\frac{1-\pi}{2}\left(p+(1-p) \sigma\left(\tilde{b}_{n}\right)\right)\right] \tilde{b}_{n}\right. \\
\left.+\frac{1}{2}\left(\frac{1-\pi}{2}\right)(1-p) \sigma\left(\tilde{b}_{n}\right)\left(1-\tilde{b}_{n}\right)\right\} \\
+\left\{-\frac{1}{2}\left[\pi\left((1-p) q \sigma\left(1-\tilde{a}_{n}\right)+p(z+(1-z) q)\right)+\frac{1-\pi}{2}\left(p+(1-p) \sigma\left(1-\tilde{a}_{n}\right)\right)\right]\left(1-\tilde{a}_{n}\right)\right. \\
\left.+\frac{1}{2}\left(\frac{1-\pi}{2}\right)(1-p) \sigma\left(1-\tilde{a}_{n}\right) \tilde{a}_{n}\right\} \\
+\left\{\pi(1-q)(1-p) z \sigma\left(\tilde{b}_{n}\right) \sigma\left(1-\tilde{a}_{n}\right)\left[\frac{1}{2}\left(-\frac{\tilde{b}_{n}}{2}+\frac{\tilde{a}_{n}}{2}\right)+\frac{1}{2}\left(\frac{1-\tilde{b}_{n}}{2}+\frac{\tilde{a}_{n}-1}{2}\right)\right]\right\},
\end{gathered}
$$

where the first two curly brackets capture case (a) in the previous paragraph, and the last curly bracket captures case (b). Now, using Lemma D1, the term inside the first curly bracket is at most 0 . By symmetry, the same remark applies to the second curly bracket. Finally, the third curly bracket is equal to $\pi(1-q)(1-p) z \sigma\left(\tilde{b}_{n}\right) \sigma\left(1-\tilde{a}_{n}\right)\left(\frac{\tilde{a}_{n}-\tilde{b}_{n}}{2}\right)$, which, since $\tilde{a}_{n} \leq \tilde{b}_{n}$, is at most 0 . So $\left(\tilde{a}_{n}, \tilde{b}_{n}\right)$ is not a profitable deviation of $\mathrm{MM} n \mathrm{U}$.

Step 3: there exists no profitable deviation of $\mathrm{MM} n \mathrm{U}$ to $\left(a_{n}, b_{n}\right) \in \Gamma$.

Suppose $\mathrm{MM} n \mathrm{U}$ deviates to $\left(\tilde{a}_{n}, \tilde{b}_{n}\right) \in \Gamma$, say $\tilde{a}_{n}=1$ and $\tilde{b}_{n} \in[l, u]$ (the other case is analogous, by symmetry). Consider first the ask side of the market: either $V=1$ or $\tilde{a}_{n} \neq \hat{a}$ with probability 1 . So the expected profit of $\mathrm{MM} n \mathrm{U}$ on the ask side of the market is at most 0 . Next, consider the bid side of the market. By virtue of (IV), (V), (D12a) and (D12b) the speculator sells and trades with $\mathrm{MM} n \mathrm{U}$ if and only if $V=0$ and:

- either $I_{S}=1$;

- or $I_{m} \wedge Z=1$ and $\tilde{b}_{n} \leq 1-a_{m}$. 
Thus MM $n$ U's expected profit on the bid side of the market may be written as

$$
\begin{aligned}
- & \frac{1}{2}\left[\pi\left(q+(1-q) z p \mathbb{P}\left(\tilde{b}_{n} \leq 1-a_{m} \mid \mathrm{MM} m \mathrm{~L}\right)\right)+\frac{1-\pi}{2}\right] \tilde{b}_{n} \\
& +\frac{1}{2}\left(\frac{1-\pi}{2}\right)\left[(1-p)+p \bar{\sigma}\left(\tilde{b}_{n}\right)\right]\left(1-\tilde{b}_{n}\right) .
\end{aligned}
$$

Conditional on $\mathrm{MM} m \mathrm{~L}, 1-a_{m}$ is distributed according to the $\operatorname{cdf} \bar{\sigma}$. Hence, using condition (C), $\mathbb{P}\left(\tilde{b}_{n} \leq 1-a_{m} \mid \mathrm{MM} m \mathrm{~L}\right)=1-\bar{\sigma}\left(\tilde{b}_{n}\right) \geq h\left(\tilde{b}_{n}\right)$. Substituting this inequality into the last highlighted expression shows that MM $n$ U's expected profit on the bid side of the market is bounded above by

$$
-\frac{1}{2}\left[\pi\left(q+(1-q) z p h\left(\tilde{b}_{n}\right)\right)+\frac{1-\pi}{2}\right] \tilde{b}_{n}+\frac{1}{2}\left(\frac{1-\pi}{2}\right)\left[(1-p)+p \bar{\sigma}\left(\tilde{b}_{n}\right)\right]\left(1-\tilde{b}_{n}\right) .
$$

By (D8), we can rewrite (D13) as

$$
-\frac{1}{2}\left[\pi\left(q+(1-q) z p h\left(\tilde{b}_{n}\right)\right)+\frac{1-\pi}{2}\right] \tilde{b}_{n}+\frac{(1-\pi)(1-u)}{4},
$$

which, by definition of $h\left(\tilde{b}_{n}\right)$, is equal to 0 . So $\left(\tilde{a}_{n}, \tilde{b}_{n}\right)$ is not a profitable deviation of $\mathrm{MM} n \mathrm{U}$.

Step 4: there exists no profitable deviation of $\mathrm{MM} n \mathrm{H}$ to $\left(a_{n}, b_{n}\right) \notin \Gamma$, with $b_{n}<a_{n}$.

Suppose $\mathrm{MM} n \mathrm{H}$ deviates to $\left(\tilde{a}_{n}, \tilde{b}_{n}\right) \notin \Gamma$, with $\tilde{b}_{n}<\tilde{a}_{n}$. Note to start with that $\mathrm{MM} n \mathrm{H}$ 's expected profit on the ask side of the market has to be non-positive. Consider next the bid side of the market. Observe that by (IV), (V), (D12c) and (D12d), the speculator never sells to $\mathrm{MM} n \mathrm{H}$. Hence, the "demand" facing $\mathrm{MM} n \mathrm{U}$ is the same as it is on the equilibrium path. In consequence, MM $n \mathrm{H}$ 's expected profit on the bid side of the market can be written like the maximand of (D8), with $b=\tilde{b}_{n}$. Yet, by virtue of Lemma D1, the maximand of (D8) is maximized when $\mathrm{MM} n \mathrm{H}$ sticks to the proposed equilibrium strategy. So $\left(\tilde{a}_{n}, \tilde{b}_{n}\right)$ is not a profitable deviation of $\mathrm{MM} n \mathrm{H}$.

Step 5: there exists no profitable deviation of $\mathrm{MM} n \mathrm{H}$ to $\left(a_{n}, b_{n}\right) \notin \Gamma$, with $a_{n} \leq b_{n}$.

Suppose $\mathrm{MM} n \mathrm{H}$ deviates to $\left(\tilde{a}_{n}, \tilde{b}_{n}\right) \notin \Gamma$, with $\tilde{a}_{n} \leq \tilde{b}_{n}$. We start by showing that $\mathrm{MM} n \mathrm{H}$ cannot make positive expected profit against the speculator. First, by virtue of (IV) and (D12c), if $I_{S} \vee\left(I_{m} \wedge Z\right)=1$ then the speculator never sells. Furthermore, it is impossible to make profit against the speculator if she buys, since $V=1$ and $\tilde{a}_{n} \leq 1$. Hence, conditional on 
$I_{S} \vee\left(I_{m} \wedge Z\right)=1, \mathrm{MM} n \mathrm{H}$ makes at most zero profit against the speculator. Next, by virtue of $(\mathrm{V}),(\mathrm{D} 12 \mathrm{e}),(\mathrm{D} 12 \mathrm{f})$ and $(\mathrm{D} 12 \mathrm{~g})$, if $I_{S} \vee I_{m}=0$ then the only case in which MM $n$ trades with the speculator is if $Z=1, \tilde{a}_{n}=\hat{a}$ and $\tilde{b}_{n}=\hat{a}$. Furthermore, in that case, by (VIII) the speculator buys and sells the asset with probabilities $\frac{1}{2}$ each. Applying (VII), the expected profit made by $\mathrm{MM} n \mathrm{H}$ against the speculator is then

$$
\frac{1}{2}\left(1-\tilde{b}_{n}\right)+\frac{1}{2}\left(\tilde{a}_{n}-1\right)=\frac{\tilde{a}_{n}-\tilde{b}_{n}}{2} .
$$

Yet $\tilde{a}_{n} \leq \tilde{b}_{n}$. Thus $\mathrm{MM} n \mathrm{H}$ makes at most zero expected profit against the speculator. The expected profit of $\mathrm{MMnH}$ is then bounded above by the expected profit made against the liquidity trader, which we can write as $\frac{1-\pi}{2}\left[p \bar{\sigma}\left(\tilde{b}_{n}\right)+(1-p) \sigma\left(\tilde{b}_{n}\right)\right]\left(1-\tilde{b}_{n}\right)+\frac{1-\pi}{2} \mathbb{P}\left(\tilde{a}_{n}=\right.$ $\hat{a})\left(\tilde{a}_{n}-1\right)$. Since the second term is non-positive, the former expression is at most equal to $\frac{1-\pi}{2}\left[p \bar{\sigma}\left(\tilde{b}_{n}\right)+(1-p) \sigma\left(\tilde{b}_{n}\right)\right]\left(1-\tilde{b}_{n}\right)$, which by (D8) is at most equal to MM $n$ H's expected profit in the proposed equilibrium. So $\left(\tilde{a}_{n}, \tilde{b}_{n}\right)$ is not a profitable deviation of $\mathrm{MM} n \mathrm{H}$.

Step 6: there exists no profitable deviation of $\mathrm{MMnH}$ to $\left(a_{n}, b_{n}\right) \in \Gamma$.

There are two possible cases. $\mathrm{MM} n \mathrm{H}$ could deviate to masquerade as $\mathrm{MM} n \mathrm{~L}$ or $\mathrm{MM} n \mathrm{H}$ could deviate to masquerade as $\mathrm{MM} n \mathrm{U}$. Suppose $\mathrm{MM} n \mathrm{H}$ deviates to masquerade as $\mathrm{MM} n \mathrm{~L}$. Then $b_{n}=0<\hat{b}$ with probability 1 . So the expected profit of $\mathrm{MM} n \mathrm{H}$ on the bid side of the market is 0 . On the other hand, since $V=1$, the profit of $\mathrm{MM} n \mathrm{H}$ on the ask side of the market is bounded above by 0 . Since sticking to his proposed equilibrium strategy yields $\mathrm{MM} n \mathrm{H}$ an expected profit of $\bar{\Pi}(p, q)>0$, deviating to masquerade as $\mathrm{MM} n \mathrm{~L}$ is therefore not a profitable deviation. Next, suppose $\mathrm{MM} n \mathrm{H}$ deviates to masquerade as $\mathrm{MM} n \mathrm{U}$. Reasoning as above, the expected profit of $\mathrm{MM} n \mathrm{H}$ on the ask side of the market is bounded above by 0 . Consider now the bid side of the market, with $b_{n}=\tilde{b}_{n} \in[0, l]$. Since $V=1$, we deduce from $(\mathrm{IV}),(\mathrm{V})$ and (D12a) that the speculator never sells. MM $n$ H's expected profit on the bid side of the market can thus be written as $\left(\frac{1-\pi}{2}\right)\left[p \bar{\sigma}\left(\tilde{b}_{n}\right)+(1-p) \sigma\left(\tilde{b}_{n}\right)\right]\left(1-\tilde{b}_{n}\right)$, which, applying Lemma D1, is bounded above by MMnH's expected profit on the bid side of the market in the proposed equilibrium. So deviating to masquerade as $\mathrm{MM} n \mathrm{U}$ is not a profitable deviation either. 
Lemma D2. Assume $p \in(0,1)$ and $q<1$. Let $\bar{\Pi}_{n}(p, q), l$, $u$ and $h(\cdot)$ be defined by (D1), (D4), (D5), and (D11) respectively. Then:

(i) for all $\varepsilon>0, p>1-\frac{2 \varepsilon}{1-\pi}$ implies $\bar{\Pi}_{n}(p, q)<\varepsilon$;

(ii) for all $\delta>0, p>1-\delta$ implies $l<\delta$ and $h(b)<0$ for all $b \in[\delta, u]$;

(iv) $1-u>\frac{1-p}{2}$.

Proof: By Lemma D1,

$$
\bar{\Pi}_{n}(p, q)=\left(\frac{1-\pi}{2}\right)(1-u)=\left(\frac{1-\pi}{2}\right)(1-p)(1-l) .
$$

Hence, $1-p<\frac{2 \varepsilon}{1-\pi}$ implies $\bar{\Pi}_{n}(p, q)<\varepsilon$, giving part (i) of the lemma. Part (iii) follows from the remark that $l<\frac{1}{2}$.

We now show part (ii) of the lemma. The denominator on the right-hand side of (D4) is minimized at $q=0$ and $z=0$, with minimum value $(2-p)(1-\pi)>1-\pi$. Hence,

$$
l \leq \frac{(1-\pi)(1-p)}{1-\pi}=1-p
$$

Pick a $\delta>0$. Then, $p>1-\delta$ implies $l<\delta$. We next show that choosing $p>1-\delta$ also implies $h(b)<0$ for all $b \in[\delta, u]$. First, rearranging (D11) gives

$$
-\frac{1}{2}\left[\pi(q+(1-q) z p h(b))+\frac{1-\pi}{2}\right] b+\frac{(1-\pi)(1-u)}{4}=0,
$$

which, by Lemma D1, we can rewrite as

$$
\left[\pi(q+(1-q) z p h(b))+\frac{1-\pi}{2}\right] b=\bar{\Pi}_{n}(p, q) .
$$

Solving for $h(b)$ gives

$$
h(b)=\frac{2 \bar{\Pi}_{n}(p, q)-b(1-\pi)-2 b \pi q}{2 b p z \pi(1-q)} .
$$

In particular,

$$
h(b) \leq \frac{1}{2 b p z \pi(1-q)}\left[2 \bar{\Pi}_{n}(p, q)-b(1-\pi)\right], \quad \forall b \in[l, u] .
$$


Now let $\varepsilon:=\frac{\delta(1-\pi)}{2}$. By part (i) of the lemma, $p>1-\frac{2 \varepsilon}{1-\pi}$ implies $\bar{\Pi}_{n}(p, q)<\varepsilon$, so, $p>1-\delta$ implies $\bar{\Pi}_{n}(p, q)<\varepsilon$. Finally, using (D14), $p>1-\delta$ implies

$$
h(b)<\frac{1}{2 b p z \pi(1-q)}[2 \varepsilon-\delta(1-\pi)]=0, \quad \forall b \in[\delta, u] .
$$

Proposition D3. There exists a function $\bar{z}()>$.0 , independent of $q$, such that a WELM trading equilibrium exists whenever $z \leq \bar{z}(p)$. Moreover, $\bar{z}(p)=1$ for $p=0$ and all $p \geq$ $\frac{\sqrt{2 \pi}}{\sqrt{2 \pi}+\sqrt{1-\pi}}$. If $q=1$, a WELM trading equilibrium exists for all values of $p$ and $z$.

Proof: We remarked at the beginning of this appendix that if $q=1$ or $p=1$ (or both) the existence of a WELM trading equilibrium then follows from the existence of a trading equilibrium in the baseline model. That $\bar{z}(p)=1$ for $p=0$ is easy to show. We assume in the rest of the proof that $p \in(0,1)$ and $q<1$.

Step 1: there exists $\bar{z}(p)>0$, independent of $q$, such that $z \leq \bar{z}(p)$ implies that a WELM trading equilibrium exists.

Define, for all $b \in[l, u], D(b):=1-\bar{\sigma}(b)-h(b)$, where $\bar{\sigma}(\cdot), l, u$ and $h(\cdot)$ are defined respectively by (D3), (D4), (D5) and (D11). Thus,

$$
D(b)=\frac{(1-\pi)(1-b)-2 \bar{\Pi}_{n}(p, q)}{(1-\pi)(1-b) p}-\frac{2 \bar{\Pi}_{n}(p, q)-b(1-\pi)-2 b \pi q}{2 b p z \pi(1-q)}, \quad \forall b \in[l, u],
$$

with $\bar{\Pi}_{n}(p, q)$ given by (D1). By Proposition D2, it suffices for our purpose to show the existence of $\bar{z}(p)>0$, independent of $q$, such that $z \leq \bar{z}(p)$ implies $D(b) \geq 0$ for all $b \in[l, u]$. First, straightforward algebra establishes that $h(l)=1$ and $\bar{\sigma}(l)=0$. Hence,

$$
D(l)=0 .
$$

Next, differentiating (D15) gives

$$
D^{\prime}(b)=\frac{\bar{\Pi}_{n}(p, q)}{p}\left(\frac{1}{b^{2} \pi(1-q) z}-\frac{2}{(1-b)^{2}(1-\pi)}\right), \quad \forall b \in[l, u] .
$$

The bracketed expression on the right-hand side of (D17) is decreasing in $b$ and increasing in 
$q$, so

$$
D^{\prime}(b) \geq \frac{\bar{\Pi}_{n}(p, q)}{p}\left[\frac{1}{u^{2} \pi z}-\frac{2}{(1-u)^{2}(1-\pi)}\right], \quad \forall b \in[l, u] .
$$

We showed in Lemma D2 that $1-u>\frac{1-p}{2}$, so the last inequality implies

$$
D^{\prime}(b) \geq \frac{\bar{\Pi}_{n}(p, q)}{p}\left[\frac{1}{\pi z}-\frac{8}{(1-p)^{2}(1-\pi)}\right], \quad \forall b \in[l, u] .
$$

The expression inside the square bracket is independent of $q$, and tends to $+\infty$ as $z$ tends to 0 . Hence, there exists $\bar{z}(p)>0$, independent of $q$, such that $z \leq \bar{z}(p)$ implies $D^{\prime}(b) \geq 0$ for all $b \in[l, u]$. Since $D(l)=0$, we obtain $D(b) \geq 0$ for all $b \in[l, u]$ whenever $z \leq \bar{z}(p)$.

Step 2: $\bar{z}(p)=1$ for all $p \geq \frac{\sqrt{2}}{\sqrt{2}+\sqrt{1-\pi}}$

By virtue of (D17),

$$
D^{\prime}(b) \geq \frac{\bar{\Pi}_{n}(p, q)}{p}\left[\frac{1}{b^{2} \pi}-\frac{2}{(1-b)^{2}(1-\pi)}\right], \quad \forall b \in[l, u] .
$$

Define

$$
\delta:=\frac{\sqrt{1-\pi}}{\sqrt{2 \pi}+\sqrt{1-\pi}} .
$$

Thus,

$$
\frac{1}{\delta^{2} \pi}=\frac{2}{(1-\delta)^{2}(1-\pi)}
$$

and, using (D19), $D^{\prime}(b) \geq 0$ for all $b \leq \delta$. By Lemma D2, $p>1-\delta$ implies $l<\delta$. So $p>1-\delta$ implies $D^{\prime}(b) \geq 0$ for all $b \in[l, \delta]$. By (D16), $p>1-\delta$ therefore implies $D(b) \geq 0$ for all $b \in[l, \delta]$. Yet, by Lemma D2, $p>1-\delta$ also implies $h(b)<0$ for all $b \in[\delta, u]$. So $p>1-\delta$ implies $D(b) \geq 0$ for all $b \in[l, u]$.

Proposition D4. A WELM trading equilibrium exists for all values of $p$ and $q$ if $z \leq$ $\frac{(1-\pi)^{2}}{8 \pi(\sqrt{2 \pi}+\sqrt{1-\pi})^{2}}$. In particular, for $(1-\pi)^{2} \geq 8 \pi(\sqrt{2 \pi}+\sqrt{1-\pi})^{2}$, a WELM trading equilibrium exists for all values of $p, q$ and $z$.

Proof: Recall: if $q=1$ or $p \in\{0,1\}$ (or both) the existence of a WELM trading equilibrium then follows from the existence of a trading equilibrium in the baseline model. We therefore 
assume in the rest of the proof that $p \in(0,1)$ and $q<1$.

Next, assume $z \leq \frac{(1-\pi)^{2}}{8 \pi(\sqrt{2 \pi}+\sqrt{1-\pi})^{2}}$. By Proposition D3, a WELM trading equilibrium exists whenever $p \geq \tilde{p}:=\frac{\sqrt{2 \pi}}{\sqrt{2 \pi}+\sqrt{1-\pi}}$. Next, let, as in the proof of Proposition D3, $D(b):=1-\bar{\sigma}(b)-$ $h(b)$. By (D18), $p<\tilde{p}$ implies

$$
D^{\prime}(b) \geq \frac{\bar{\Pi}_{n}(p, q)}{p}\left[\frac{1}{\pi z}-\frac{8}{(1-\tilde{p})^{2}(1-\pi)}\right], \quad \forall b \in[l, u] .
$$

Yet,

$$
\frac{1}{\pi z}-\frac{8}{(1-\tilde{p})^{2}(1-\pi)} \geq 0 \Longleftrightarrow z \leq \frac{(1-\pi)^{2}}{8 \pi(\sqrt{2 \pi}+\sqrt{1-\pi})^{2}} .
$$

Thus, $p<\tilde{p}$ implies $D^{\prime}(b) \geq 0$ for all $b \in[l, u]$. Since $D(l)=0$, we obtain $D(b) \geq 0$ for all $b \in[l, u]$ whenever $p<\tilde{p}$. By Proposition D2, a WELM trading equilibrium therefore exists for all $p<\tilde{p}$. 


\section{References}

Albanesi, S. and Rindi, B. (2000) The quality of the Italian treasury bond market, asymmetric information and transaction costs, Annales d'Economie et de Statistique, 60, 1-19.

Anand, A. and Subrahmanyam, A. (2006) Information and the intermediary: Are market intermediaries informed traders in electronic markets?, Available at SSRN 700864.

Atakan, A. and Ekmekci, M. (2019) Price discovery with costly competitive bidding, mimeo.

Bao, J., Pan, J. and Wang, J. (2011) The illiquidity of corporate bonds, Journal of Finance, 66, 911-946.

Bloomfield, R. and O'Hara, M. (2000) Can transparent markets survive?, Journal of Financial Economics, 55, 425-459.

Bolton, P. and Faure-Grimaud, A. (2010) Satisficing contracts, Review of Economic Studies, 77, 937-971.

Boulatov, A. and George, T. J. (2013) Hidden and displayed liquidity in securities markets with informed liquidity providers, Review of Financial Studies, 26, 2096-2137.

Budish, E., Cramton, P. and Shim, J. (2015) The high-frequency trading arms race: Frequent batch auctions as a market design response, Quarterly Journal of Economics, 130, 15471621.

Chamley, C. (2007) Complementarities in information acquisition with short-term trades, Theoretical Economics, 2, 441-467.

Chen, L., Lesmond, D. A. and Wei, J. (2007) Corporate yield spreads and bond liquidity, Journal of Finance, 62, 119-149.

Clark-Joseph, A. D., Ye, M. and Zi, C. (2017) Designated market makers still matter: Evidence from two natural experiments, Journal of Financial Economics, 126, 652-667.

Covrig, V. and Melvin, M. (2002) Asymmetric information and price discovery in the FX market: does Tokyo know more about the yen?, Journal of Empirical Finance, 9, 271-285.

Dang, T. V. (2008) Bargaining with endogenous information, Journal of Economic Theory, 140, 339-354. 
de Frutos, M. A. and Manzano, C. (2005) Trade disclosure and price dispersion, Journal of Financial Markets, 8, 183-216.

Edwards, A. K., Harris, L. E. and Piwowar, M. S. (2007) Corporate bond market transaction costs and transparency, Journal of Finance, 62, 1421-1451.

Engelbrecht-Wiggans, R., Milgrom, P. R. and Weber, R. J. (1983) Competitive bidding and proprietary information, Journal of Mathematical Economics, 11, 161-169.

Foucault, T. (1999) Order flow composition and trading costs in a dynamic limit order market, Journal of Financial markets, 2, 99-134.

Foucault, T., Pagano, M. and Röell, A. (2013) Market liquidity: theory, evidence, and policy, Oxford University Press.

Glosten, L. R. and Milgrom, P. R. (1985) Bid, ask and transaction prices in a specialist market with heterogeneously informed traders, Journal of Financial Economics, 14, 71-100.

Grossman, S. J. and Stiglitz, J. E. (1980) On the impossibility of informationally efficient markets, American Economic Review, 70, 393-408.

Hasbrouck, J. (2007) Empirical market microstructure: The institutions, economics, and econometrics of securities trading, Oxford University Press.

Huang, R. D. (2002) The quality of ECN and Nasdaq market maker quotes, Journal of Finance, 57, 1285-1319.

Kaniel, R. and Liu, H. (2006) So what orders do informed traders use?, Journal of Business, 79, 1867-1913.

Leach, J. C. and Madhavan, A. N. (1993) Price experimentation and security market structure, Review of Financial Studies, 6, 375-404.

Lee, T. K. (1984) Incomplete information, high-low bidding and public information in first price auctions, Management Science, 30, 1490-1496.

Li, X. and Heidle, H. G. (2004) Information leakage and opportunistic behavior before analyst recommendations: An analysis of the quoting behavior of Nasdaq market makers, mimeo. 
Manaster, S. and Mann, S. C. (1996) Life in the pits: Competitive market making and inventory control, Review of Financial Studies, 9, 953-975.

Massa, M. and Simonov, A. (2003) Reputation and interdealer trading: A microstructure analysis of the treasury bond market, Journal of Financial Markets, 6, 99-141.

Menkveld, A. J. (2013) High frequency trading and the new market makers, Journal of Financial Markets, 16, 712-740.

Menkveld, A. J. (2016) The economics of high-frequency trading: Taking stock, Annual Review of Financial Economics, 8, 1-24.

Milgrom, P. R. (1981) Rational expectations, information acquisition, and competitive bidding, Econometrica, 49, 921-943.

Persico, N. (2000) Information acquisition in auctions, Econometrica, 68, 135-148.

Sapp, S. G. (2002) Price leadership in the spot foreign exchange market, Journal of Financial and Quantitative Analysis, 37, 425-448.

Stoll, H. R. (1978) The pricing of security dealer services: An empirical study of NASDAQ stocks, Journal of Finance, 33, 1153-1172.

Tirole, J. (2009) Cognition and incomplete contracts, American Economic Review, 99, 265-94.

Tirole, J. (2015) Cognition-intensive contracting, mimeo.

Valseth, S. (2013) Price discovery in government bond markets, Journal of Financial Markets, 16, 127-151.

Verrecchia, R. E. (1982) Information acquisition in a noisy rational expectations economy, Econometrica, 50, 1415-1430.

Wilson, R. B. (1967) Competitive bidding with asymmetric information, Management Science, 13, 816-820. 